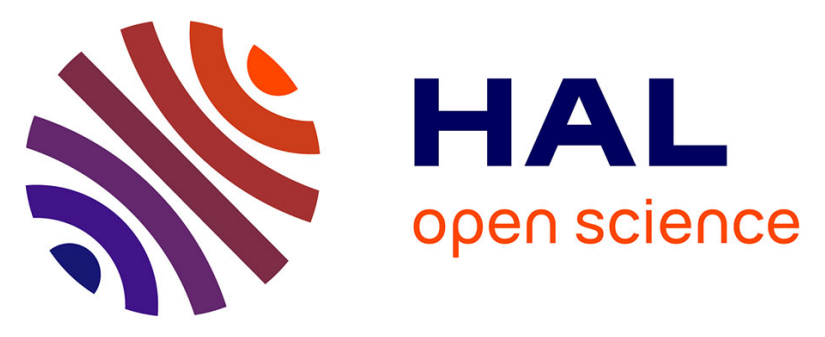

\title{
Three dimensional experimental and numerical multiscale analysis of a fatigue crack
}

Johann Rannou, Nathalie Limodin, Julien Réthoré, Anthony Gravouil, Wolfgang Ludwig, Marie-Christine Baietto, Jean-Yves Buffiere, Alain Combescure, François Hild, Stéphane Roux

\section{To cite this version:}

Johann Rannou, Nathalie Limodin, Julien Réthoré, Anthony Gravouil, Wolfgang Ludwig, et al.. Three dimensional experimental and numerical multiscale analysis of a fatigue crack. Computer Methods in Applied Mechanics and Engineering, 2010, 199, pp.1307-1325. 10.1016/j.cma.2009.09.013 . hal00430486v1

\section{HAL Id: hal-00430486 \\ https://hal.science/hal-00430486v1}

Submitted on 7 Nov 2009 (v1), last revised 15 Sep 2010 (v2)

HAL is a multi-disciplinary open access archive for the deposit and dissemination of scientific research documents, whether they are published or not. The documents may come from teaching and research institutions in France or abroad, or from public or private research centers.
L'archive ouverte pluridisciplinaire HAL, est destinée au dépôt et à la diffusion de documents scientifiques de niveau recherche, publiés ou non, émanant des établissements d'enseignement et de recherche français ou étrangers, des laboratoires publics ou privés. 


\title{
Three dimensional experimental and
}

\section{numerical multiscale analysis of a fatigue}

\section{crack}

Johann Rannou, ${ }^{a}$ Nathalie Limodin, ${ }^{b}$ Julien Réthoré, ${ }^{a}$ Anthony Gravouil, ${ }^{a}$ Wolfgang Ludwig, ${ }^{b, c}$

Marie-Christine Baïetto-Dubourg, ${ }^{a}$ Jean-Yves Buffière, ${ }^{b}$

Alain Combescure, ${ }^{a}$ François Hild, ${ }^{d}$ Stéphane Roux ${ }^{d}$

(a): Laboratoire de Mécanique des Contacts et des Structures (LaMCoS),

$$
\text { INSA-Lyon / UMR CNRS } 5259
$$

20 avenue Albert Einstein, F-69621 Villeurbanne, France

(b): Laboratoire Matériaux, Ingénierie et Sciences (MATEIS),

INSA-Lyon / UMR CNRS 5510

7, avenue Jean Capelle, F-69621 Villeurbanne, France

(c): European Synchrotron Radiation Facility/Experimental Division (ESRF), BP 220, 6 rue J. Horowitz, F-38043 Grenoble, Cedex, France

(d): Laboratoire de Mécanique et Technologie (LMT-Cachan)

ENS Cachan / CNRS / UPMC / PRES UniverSud Paris

61 Avenue du Président Wilson, F-94235 Cachan Cedex, France

\begin{abstract}
A full three dimensional study of a fatigue crack in cast iron is presented. This analysis involves combining various tools, namely, Synchrotron X-ray microtomography
\end{abstract}


of an in situ experiment, image acquisition and treatment, 3D volume correlation to measure 3D displacement fields, extraction of the crack geometry, extended digital image correlation to account for the crack displacement discontinuity, crack modeling in an elastic material exploiting the actual crack geometry, and finally estimation of stress intensity factors. All these different tasks are based on specific multiscale approaches.

Key words: 3D image correlation, fatigue crack, X-ray microtomography, multiscale analyses, SIF estimates, level sets, multi-grid, X-FEM

Manuscript submitted for publication in CMAME, Special issue on "ADVANCES IN MULTISCALE MODELING AND ANALYSIS METHODS IN APPLICATIONS TO SOLIDS AND FLUIDS".

Email addresses: johann.rannou@insa-lyon.fr (Johann Rannou, $\left.{ }^{a}\right)$, nathalie.limodin@insa-lyon.fr (Nathalie Limodin, $\left.{ }^{b}\right)$, julien.rethore@insa-lyon.fr (Julien Réthoré, $\left.{ }^{a}\right)$, anthony.gravouil@insa-lyon.fr (Anthony Gravouil, ${ }^{a}$ ), ludwig@esrf.fr (Wolfgang Ludwig, ${ }^{b, c}$ ), marie-christine.baietto@insa-lyon.fr (Marie-Christine Baïetto-Dubourg, ${ }^{a}$ ), jean-yves.buffiere@insa-lyon.fr $\left(\right.$ Jean-Yves Buffière $^{b}{ }^{b}$, alain.combescure@insa-lyon.fr (Alain Combescure, ${ }^{a}$ ), francois.hild@lmt.ens-cachan.fr (François Hild, $\left.{ }^{d}\right)$, stephane.roux@lmt.ens-cachan.fr (Stéphane Roux $\left.{ }^{d}\right)$. 


\section{Introduction}

To analyze the long-term reliability of structures, there is a need for robust and validated 3D crack propagation models and numerical procedures. This effort involves a number of specific challenges on the numerical modeling side because of the very demanding cost of three dimensional approaches. Validation itself appears to be even more challenging as it requires a full three dimensional comparison between models and experiments, and the latter requires three dimensional investigation instruments. The present study illustrates one of the routes that can be followed to achieve a direct link between experimental and computational mechanics $[1-5]$.

Fatigue cracks are three dimensional objects in essence, but the lack of 3D data about crack propagation in optically opaque materials has made necessary the development of simplified 2D fatigue models. However, even for one of the simplest (and widely used) crack geometry like a through crack in a plate specimen, 2D measurements via an optical microscope only give access to the crack tip position on a free surface, i.e., schematically in plane stress conditions while the crack propagates faster inside the specimen, i.e., closer to plane strain conditions. The phenomenon of crack closure [6] can be used to account for the differences between surface and bulk propagation. It is usually assessed by considering surface observations, for instance by using displacement fields $[7,8]$. However, it was shown that this effect is mainly active in the vicinity of free surfaces [9]. This last result was obtained by coupling X-ray microtomography with X-FEM simulations relying upon a visual determination of the crack front.

In the present work, it is proposed to integrate even more the experimen- 
tal analysis and the numerical tools by combining the following procedures (Figure 1):

- X-ray tomography to get 3D pictures of in situ tests in, say, a synchrotron facility. By post-processing them, one may get, for instance, a first estimate of the morphology of the cracked surface,

- volume correlation to measure displacement fields whose kinematic basis is consistent with experiments on cracked samples and subsequent X-FEM analyses,

- description of the crack shape by a level set modeling,

- multi-grid X-FEM simulation based on the actual crack shape determination,

- extraction procedures to identify stress intensity factors, either from the correlation (i.e., measurement) procedure or from an elastic modeling.

X-Ray Computed MicroTomography (XCMT) is a very powerful way of imaging material microstructures in a non destructive manner. By reconstruction from $2 \mathrm{D}$ pictures (radiographs), it allows for a $3 \mathrm{D}$ visualization of various phases of materials $[10,11]$. One has also access, for instance, to microstructural changes during solidification of alloys [12], to microstructural details during sintering of steel powders $[13,14]$, to damage mechanisms in the bulk of particulate composites $[15,16]$ or to the structure of cellular materials (either metallic or polymeric foams) and strains $[17,18]$. These actual microstructures may be further processed using finite element tools [19].

In the following, 3D Digital Image Correlation (3D-DIC) and tomographic techniques are applied to measure displacement fields in nodular graphite cast iron when cracked (Section 2). This step is achieved by resorting to correlation techniques. DIC is a technique that consists in measuring displacement fields 


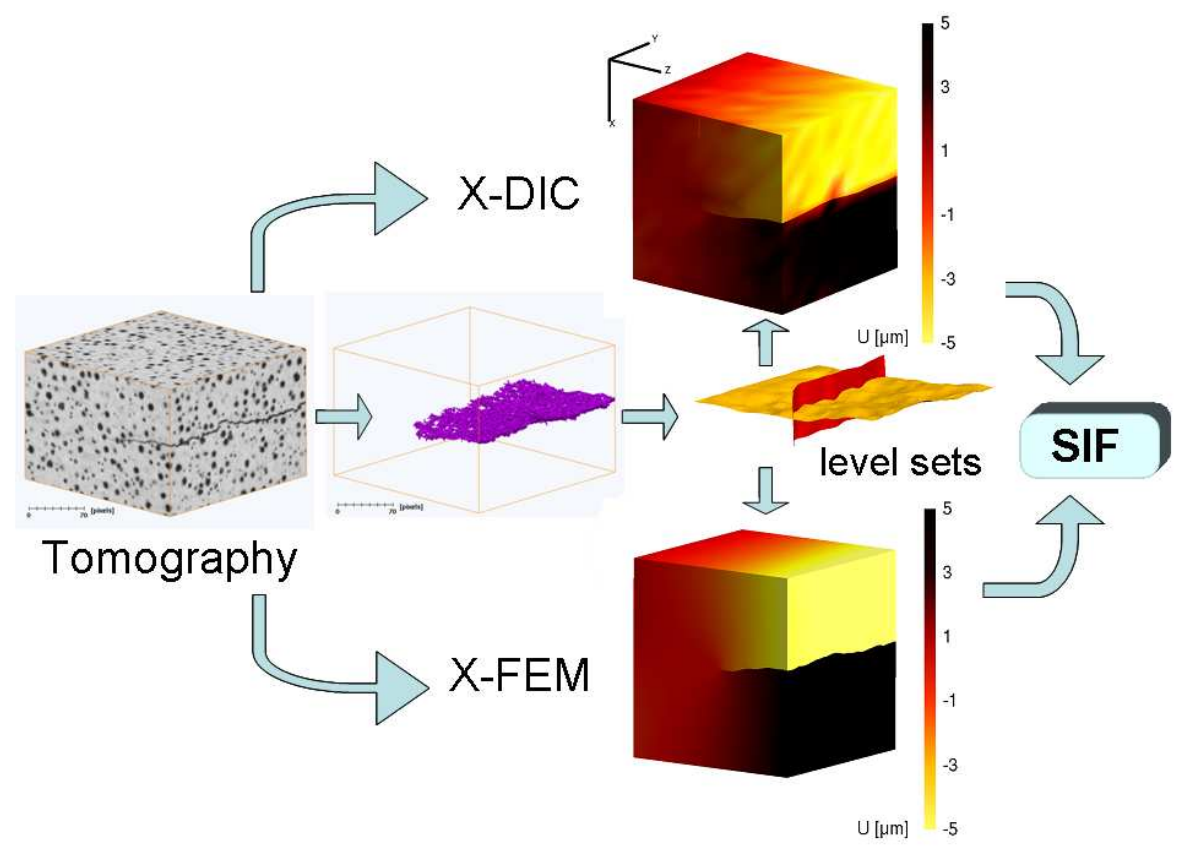

Fig. 1. Schematic view of the methods used in the present paper for determining stress intensity factors along the front of a crack imaged by computed tomography. based on image pairs of the same specimen at different stages of loading [20]. The displacement field is computed so that the image of the loaded sample is matched to the reference image when voxel locations are corrected for by the displacement field. The most commonly used correlation algorithms consist in matching locally small zones of interest in a sequence of pictures to determine local displacement components [21]. The same type of hypotheses are made in three-dimensional algorithms [22-25]. This study reports on a different algorithm, namely a Galerkin approach to DIC that has been shown to be more performing in two dimensions when compared to traditional DIC algorithms [26, 27]. It has been extended to three dimensions for continuous displacements [28]. Strain localization could be analyzed in a solid foam. Section 3 presents the application of this DIC algorithm to tomographic images.

The next step is to further extend the finite element shape function description 
of the kinematics to an enriched basis as used in X-FEM simulations [32] and previously performed to analyze 2D cases $[33,34]$. The enriched kinematics, in the spirit of X-FEM, needs a precise description of the crack geometry. Image analysis techniques (e.g., binarization, skeletonization, extraction of connected component) have been successfully used for rather homogeneous materials [9]. In the present case, the heterogeneous microstructure, which is very helpful in terms of image correlation, becomes a strong obstacle to resort to such tools on 3D images. However, exploiting image correlation residuals offers a very valuable field from which the crack surface may be extracted. A direct processing of this field, and a new automatic extraction algorithm are presented in Section 4.

Section 5 is devoted to the presentation of the extended DIC analysis, as previously performed to analyze $2 \mathrm{D}$ cases $[33,34]$ and here extended to the three dimensional case. After introducing the theoretical framework, a priori performances are studied both to choose the appropriate size of the elements and to provide a comparison with non-extended DIC. Last, the displacement field obtained from the above technique based on the actual crack surface geometry is presented.

In a third step, the combination of XCMT and 3D digital image correlation is completed with X-FEM simulations to improve the understanding of 3D crack growth laws. This numerical model is the combination of an external finite difference mesh (to describe the crack shape in the area of interest), an X-FEM mesh (i.e., crack discontinuity and asymptotic behavior close to the crack front), a local multi-grid strategy to capture the possible scale effects with a high efficiency in terms of CPU time and numerical optimization (Section 6).

From the displacement field obtained by the previous analyses, it is possible 
to determine stress intensity factors. In $2 \mathrm{D}$ cases, the first route is a least squares minimization between the measured displacement field and its analytic asymptotic development around the crack tip [35]. The same type of fields can be implemented in an integrated DIC (I-DIC) algorithm [8, 36]. A second route, which is followed in the present study, consists in using an accurate domain integral in order to compute stress intensity factors along the crack front (both in the bulk or close to the free surfaces [37]). This is an extension over previous works that applied interaction integrals for post-processing DIC measurements $[38,39]$. In parallel, the local multi-grid X-FEM modeling of

the real crack allows one to compute the displacement and stress fields in the bulk. The very same domain integral tool is used to estimate stress intensity factors along the front (Section 7).

\section{System under study}

\subsection{Nodular graphite cast iron}

The studied material is a widely used ferritic cast iron whose composition is $3.65 \mathrm{wt} \% \mathrm{C}, 3.2 \mathrm{wt} \% \mathrm{Si}, 0.04 \mathrm{wt} \% \mathrm{Mg},<0.1 \mathrm{wt} \% \mathrm{Mn},<0.005 \mathrm{wt} \% \mathrm{~S}$, $0.02 \mathrm{wt} \% \mathrm{P}$. After casting and subsequent heat treatment (ferritization at $880^{\circ} \mathrm{C}$ followed by air cooling) the material microstructure consists of a ferritic matrix (98 wt\% ferrite and 2 wt\% perlite) containing a homogeneous distribution of nearly spherical graphite nodules (volume fraction: $13 \%$, average internodule distance: $50 \mu \mathrm{m}$, average nodule diameter: $50 \mu \mathrm{m}$ ). The large change in atomic number between carbon (nodules) and iron (matrix) gives a strong X-ray attenuation contrast on the radiographs recorded during to- 
mographic scans. The nodules are therefore easily imaged by tomography and used as natural markers for image correlation in the 3D reconstructed images. The nodule size is large enough to allow for a relatively low voxel size to be used for tomography. This also enables one to observe millimetric samples, the only limitation for the sample size being the overall attenuation of the material that has to allow for, at least, a $10 \%$ transmission of the incoming X-ray beam. The fatigue crack interactions with the microstructure gives a roughness of the crack path of the order of the grain / nodule size, which is small enough to allow for a macroscopic modeling. The values of the material Young's modulus and yield stress are $175 \mathrm{GPa}$ and $315 \mathrm{MPa}$, respectively. Poisson's ratio is equal to 0.3 .

\subsection{Sample preparation}

Notched hourglass fatigue samples with a $6 \times 4 \mathrm{~mm}^{2}$ rectangular cross section were spark cut from the as-cast material. After mechanical polishing of the sample flat faces, crack initiation at the root of the notch (radius of curvature: $170 \mu \mathrm{m})$ is monitored in situ by optical microscopy during constant amplitude cyclic loading (load ratio $R=0.2$ ). Once a crack is initiated, a load shedding procedure is applied to achieve crack growth with a constant plastic zone size of the order of $200 \mu \mathrm{m}$. When the crack size is of the order of $1.5 \mathrm{~mm}$, the fatigue test is stopped. The cross section of the cracked fatigue sample is too large to perform tomography, hence, two smaller dogbone samples with a square area of $1.6 \times 1.6 \mathrm{~mm}^{2}$ containing the fatigue crack tip are spark cut within the $4 \mathrm{~mm}$-thick fatigue sample as shown in Figure 2. One flat face of the tomography sample therefore corresponds to one flat face of the initial fatigue sample (which to a certain extent can be considered as in plane stress) 


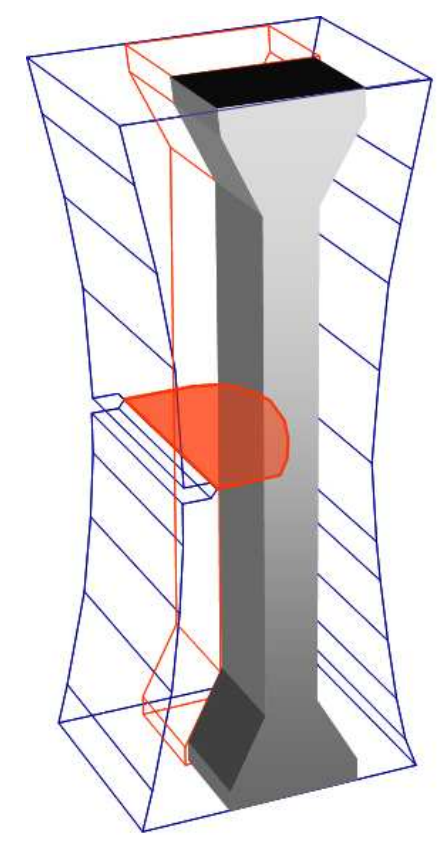

Fig. 2. Schematic view of the extraction of the specimen used for tomography from the larger one used for fatigue pre-cracking.

while the other one corresponds to the interior of the initial sample (closer to plane strain conditions) during cycling.

\subsection{X-ray tomography}

The experiment reported herein was performed at the European Synchrotron Radiation Facility (ESRF) in Grenoble (France) on Beamline ID19. An almost monochromatic X-ray beam $(\Delta \lambda / \lambda \approx 1 \%)$ having a photon energy of $60 \mathrm{keV}$ traverses the cast iron sample giving an incident-to-transmitted intensity ratio of at least $10 \%$. The synchrotron beam is parallel so that the final voxel size depends only on the optics used. To obtain a 3D image of the specimen in the vicinity of the crack, six hundred radiographs (referred to as scan) were recorded while the sample was rotating over $180^{\circ}$ along a vertical axis. A negligible amount of phase-contrast [40] was observed on the radiographs with 
the conditions of observation used (specimen / detector distance $\sim 70 \mathrm{~mm}$ ). A Fast Readout Low Noise (FReLoN) 14-bit CCD camera with a resolution of $2048 \times 2048$ pixels was used [41]. The time required to acquire each image was set to $3 \mathrm{~s}$, resulting in a total scan time of about 42 minutes.

A specially designed in situ fatigue testing machine that allows for loading and cycling (up to a frequency of $50 \mathrm{~Hz}$ ) of the specimen during scans [42] was used. At the beginning of the experiment, the specimen was loaded in four steps (30\% increments) to a maximum load of about $190 \mathrm{~N}$, corresponding to a maximum stress intensity factor slightly less than the value used for precracking the specimen. Four tomographic scans were acquired during loading and one scan during unloading. The specimen was then cycled at a load ratio of 0.1 by keeping the same maximum load. Scans were recorded at maximum load every 5,000 cycles. When significant crack advance was detected, the loading-unloading sequences were repeated, i.e., at 45,000 and 49,000 cycles.

Reconstruction of the tomographic data was performed with a conventional filtered back-projection algorithm [43]. It provides a 3D image with a 32-bit dynamic range that is proportional to the local attenuation coefficient. Then, the 32-bit image is re-encoded onto an 8-bit range to reduce data size and computing time. The final region of interest is focused on the crack and it has dimensions of $340 \times 340 \times 512$ voxels, i.e., with a voxel size of $5.06 \mu \mathrm{m}$ in the reconstructed images, $1.72 \times 1.72 \times 2.59 \mathrm{~mm}^{3}$.

\section{3D Digital Image Correlation}

In this section, an eXtended 3D Digital Image Correlation (X3D-DIC) technique is presented. Although DIC is now a rather mature field commonly used 
in fluid mechanics where it is known under the acronym of PIV, for Particle Image Velocimetry, and more and more popular in solid mechanics, full 3D displacement field applications are still scarce. The limiting point is the acquisition of three dimensional images of a specimen. This excludes many fluid mechanics applications, and requires a dedicated sophisticated lab equipment (such as an X-ray tomograph or Nuclear Magnetic Resonance Imaging (MRI) devices) or the use of large scale equipment such as a synchrotron beamline (as used in the present study). However, more and more applications appear, and the complete three dimensional description of the kinematics will prompt more of more frequent use of such approaches.

The reconstructed pictures themselves may be used to evaluate displacements. A first technique consists in tracking markers (e.g., particles). Strain uncertainties of the order of $10^{-2}$ were reported [44]. When image processing techniques based upon correlation algorithms are used, strain uncertainties of the order of $10^{-3}$ are achieved [22, 24]. A major field of application concerns biomechanical studies $[45,46]$. Other studies were devoted to micromechanical analyses of strain fields in heterogeneous materials [13, 24] and strain localization [47]. Cellular materials have also been studied [17, 18], and 2D [48] or 3D [28] strain measurements were performed.

A different approach to classical DIC was proposed recently that allows one to decompose the displacement field onto an arbitrary kinematic basis, enabling for a pixelwise consideration of the displacement field rather than a zonal average of the displacement field into its mean translational components [26]. This technique has been applied essentially to 2D problems, although studies have been published on 3D foam compression [28], and on stone wool compression [49]. The former reference gives most details on the algorithm, 
and only a brief outlook is proposed in the following subsection. The second subsection will detail eXtended DIC where, paralleling the eXtended Finite Element Method (X-FEM), discontinuity enrichments are added to the chosen functional basis to describe the presence of cracks more accurately.

\subsection{D Digital Image Correlation}

A 3D XCMT image is represented as a scalar function of the spatial coordinates, $f(x, y, z)=f(\boldsymbol{x})$ whose value indicates the local gray level. Digital image correlation is based on the comparison of image pairs, $f(\boldsymbol{x})$ and $g(\boldsymbol{x})$, the first one being referred to as the "reference image," whilst the second one is the "deformed image." A complete experiment requires a time series that may contain from one to ten or more pairs. For small displacement amplitudes, DIC analyses are usually carried out between the first and the $n^{\text {th }}$ image. However, large displacements may require chaining successive analyses of consecutive pairs (the $n^{\text {th }}$ image being considered as the reference, and compared to the $(n+1)^{\text {th }}$ image), in order to reconstruct the total displacement field [50]. In the treated example, a direct analysis (i.e., with no updating of the reference image) revealed to be sufficient.

The displacement field, $\boldsymbol{u}(\boldsymbol{x})$, is identified from the hypothesis that the texture of one image is simply convected by the displacement field so that the so-called brightness conservation reads

$$
f(\boldsymbol{x}+\boldsymbol{u}(\boldsymbol{x}))=g(\boldsymbol{x})
$$

thereby relating the reference and deformed images. This hypothesis cannot be fulfilled for each voxel, and hence a weaker form is preferred. Moreover, the two images do not contain enough information to determine the displacement 
field independently for each voxel (i.e., it is an ill-posed problem). Hence, a regularization has to be proposed based on a restricted space of displacement functions. A basis of this subspace is denoted by $\boldsymbol{\varphi}_{n}(\boldsymbol{x})$, so that the projection of the actual displacement field is searched for in this space as

$$
\boldsymbol{u}(\boldsymbol{x})=\sum a_{n} \boldsymbol{\varphi}_{n}(\boldsymbol{x})
$$

The weak brightness conservation is written as the minimization of the objective function $\mathcal{R}$

$$
\mathcal{R}=\iiint r^{2}(\boldsymbol{x}) \mathrm{d} \boldsymbol{x}
$$

where

$$
r(\boldsymbol{x}) \equiv f\left(\boldsymbol{x}+\sum a_{n} \boldsymbol{\varphi}_{n}(\boldsymbol{x})\right)-g(\boldsymbol{x})
$$

It is proposed to solve this minimization problem through linearizations of $r(\boldsymbol{x})$. This leads to the following system

$$
M A=B
$$

where $\boldsymbol{A}$ is the column vector of unknowns $A_{i}=a_{i}$,

$$
M_{i j}=\iiint\left(\nabla f(\boldsymbol{x}) \cdot \boldsymbol{\varphi}_{i}(\boldsymbol{x})\right)\left(\boldsymbol{\nabla} f(\boldsymbol{x}) \cdot \boldsymbol{\varphi}_{j}(\boldsymbol{x})\right) \mathrm{d} \boldsymbol{x}
$$

and

$$
B_{i}=\iiint(g(\boldsymbol{x})-f(\boldsymbol{x}))\left(\boldsymbol{\nabla} f(\boldsymbol{x}) \cdot \boldsymbol{\varphi}_{i}(\boldsymbol{x})\right) \mathrm{d} \boldsymbol{x}
$$

As such this procedure unfortunately does not provide the actual displacement because linearization is often illegitimate as compared to the amplitude of the displacement. For arbitrary large displacement amplitudes, a crowding of secondary local minima appears, and without a priori determination of the displacement, chances are large to be trapped in such a secondary minimum.

To circumvent this difficulty, the following multiscale approach reveals extremely efficient. Both images are coarse-grained by summing up gray-levels 
of $2 \times 2 \times 2$ groups of voxels, used as a definition of the "super-voxel" of the coarse image. This coarsening is applied recursively three to five times. The result is smaller images where the displacement amplitude becomes smaller as well. Therefore, the linearization becomes justified and hence a proper convergence to the actual displacement field is achieved. However, the accuracy of the displacement is poor because small scale details of the image were erased in the coarsening. Thus, it is proposed to first carry out the analysis at the coarser level. Then, the determined displacement field is used to correct the deformed image, and hence, the remaining displacement field to determine is expected to be of much lower amplitude. The next order can thus be analyzed, and displacements accounted for, recursively down to the original image.

It is important to note that the matrices $\boldsymbol{M}$ (one per coarsening level) remain unchanged during the identification of the displacement. Only the deformed image, and hence the second member $\boldsymbol{B}$ is updated at each step of the algorithm. In three dimensions, the coarser images are smaller by a factor of 8 at each generation. Therefore the memory and time requirements are very modest as compared to the final step. Let us also emphasize the fact that the minimization procedure naturally provides a global evaluation of the quality of the matching of both images, through the value of $\mathcal{R}$ at the final stage of the computation. More importantly, this quality factor is the spatial integral of a residual density $r$. Therefore, a map of residual discrepancies is also obtained. This map may reveal regions where the measured displacement is inaccurate or unadapted. In the sequel, it will be shown that this information is very useful, and may be utilized for the analysis of the crack geometry.

At this stage, the type of displacement basis functions to be used was not discussed. In fact, very few constraints exist on this choice, and hence a specific 
set of functions can be tailored to the problem at hand. Without any a priori information on the kinematics, a very common choice is to use finite-element shape functions, so that an interface with a subsequent numerical approach is performed by using the same discretization, i.e., without any information loss. The most natural finite element type is the 8-node trilinear cube element (C8), which is suited to the computed tomographic image format defined over a Cartesian regular grid [28]. This description will be refined in the sequel.

\subsection{DIC Results}

As one applies the correlation algorithm to image pairs, one has to choose the size of the elements, i.e. the number of voxels in the cubes that constitute the C8 elements. This size selection is an important ingredient that will affect the quality of the determination. The larger the element size, the more accurate the measure of the displacements as many voxels will contribute. However, large elements will not be able to capture strain inhomogeneities at a small scale. Thus a compromise has to be found. To quantify this property, it is important to proceed first with an a priori determination of errors and uncertainties. The discussion of such performances is differed to Section 5.2, where this issue will motivate the use of an extension of DIC to discontinuous enrichments.

In this first analysis, we chose a small element size of $16 \times 16 \times 16$ voxels. Figure 3 shows the three components of the displacement field as obtained at the maximum load level. Note that the mean rigid body motion has first been determined from the raw displacement field and subtracted off. A rather clear discontinuity is visible on the $U$ component, although by construction, the displacement discontinuity is spread over one element at least. The amplitude 
of the discontinuity (at the edge where it is the larger) is less than $10 \mu \mathrm{m}$ (less than 2-voxel size). The other components do not reveal a clear discontinuity although the location of the crack can also be guessed from those fields.

It is worth noting that these fields are noisy, as they show fluctuations at the element scale that do not correspond to the actual displacement. This is due to the choice of a small element size, so that the location of the discontinuity could be more precisely pinpointed. One will see in the sequel that if the crack discontinuity is described by a suitable enrichment, then larger elements can be used, and a lower noise level will result. One will not make any further use of these results, as an extension of DIC with an enriched description is proposed in Section 5. However, in order to proceed, one needs an accurate determination of the crack surface discontinuity. The following section shows that the residual map of this first DIC analysis will reveal to be a very precious tool for this purpose.

\section{Crack geometry identification}

For the mechanical analysis of a crack, and even for a proper description of the displacement field, it is important to have access to a faithful description of the crack surface geometry. This section presents two independent approaches to meet this objective. The first one is based on 3D image analysis tools, which have revealed very powerful to analyze cracks from tomographic images [9]. However, in the present case, it happens that the graphite nodules have a gray level that is comparable with that of the crack itself. Hence a partition between those two types of objects is quite hazardous. The result of this analysis is however a conservative estimate of the crack geometry (plus some 

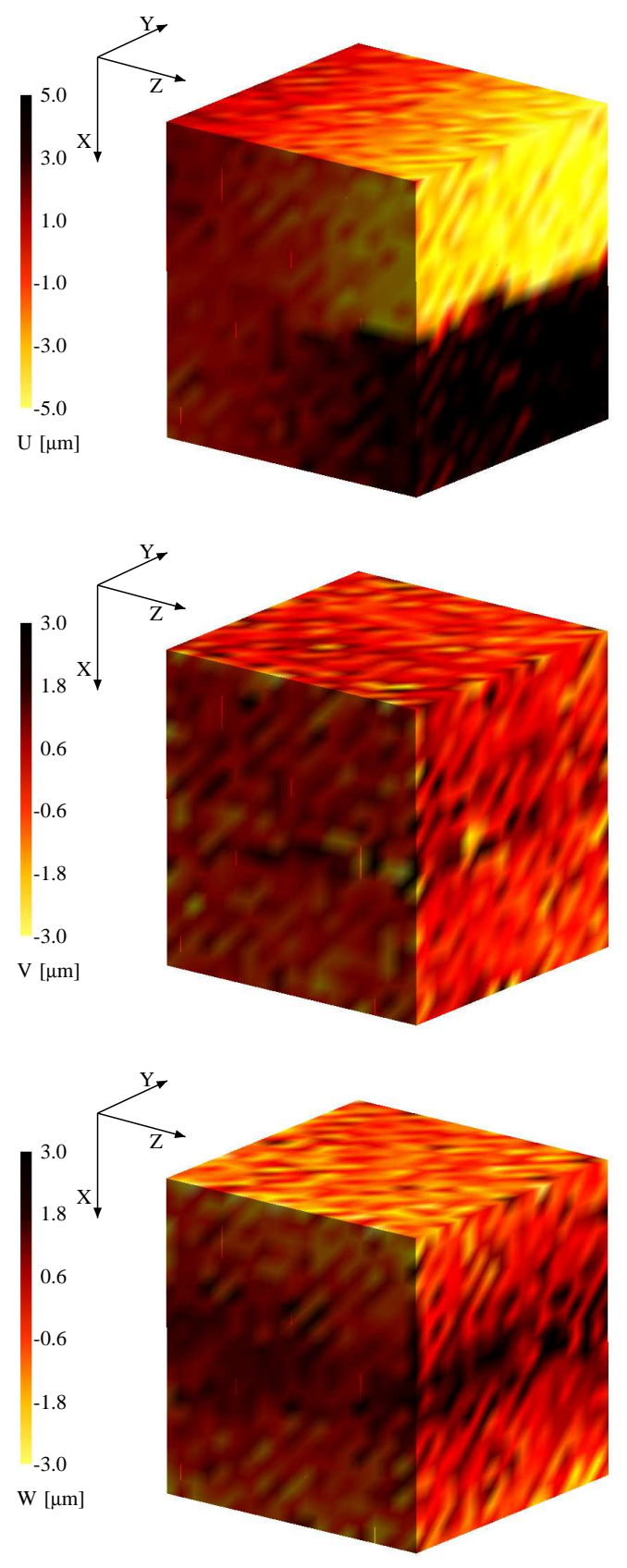

Fig. 3. The three components of the displacement field (in micrometers) for the maximum load level after 45,000 cycles with a C8 approach. The rigid body motion was removed from those fields. The volume shown in this figure is a cube whose edge is $1.46-\mathrm{mm}$ (or 288-voxel) long. 
attached nodules) from a single image, namely that with the largest load level. The second approach is based on the residual field from the image correlation analysis. A specific strategy has been conceived to extract the crack surface from this map.

\subsection{Image analysis}

The easiest way for obtaining a 3D image of a fatigue crack in the studied sample is by thresholding the 3D image gray levels. In cast iron however, the X-ray attenuation coefficient of graphite nodules is close to that of the air present in the crack so that a simple binarization based on a gray level threshold will keep the nodules in the binary image as illustrated in Figure 4.

To circumvent this problem, the error map obtained from a DIC analysis was thresholded to separate the error due to the discontinuity (i.e., the crack) from that due to tomography artifacts or computation error. A multiscale C8-DIC algorithm was used to compute the displacement fields and the corresponding residual fields. The image acquired at minimum load ( $14 \mathrm{~N}$ in this case) during the first cycle was chosen as the reference of the computation. Elements of size $16 \times 16 \times 16$ voxels, i.e., about $81 \times 81 \times 81 \mu \mathrm{m}^{3}$, resulted in a mean error of $2 \times 10^{-3}$ voxel and a standard uncertainty of $7 \times 10^{-2}$ voxel (as discussed below).

The error map is digitized on an 8-bit color map and thresholded using a region growing algorithm implemented in the 3D image analysis software Amira [52]; the gray levels from 136 to 255 were ascribed to the crack. Figure 5 shows the crack surface obtained by this method at the maximum load level, i.e., 


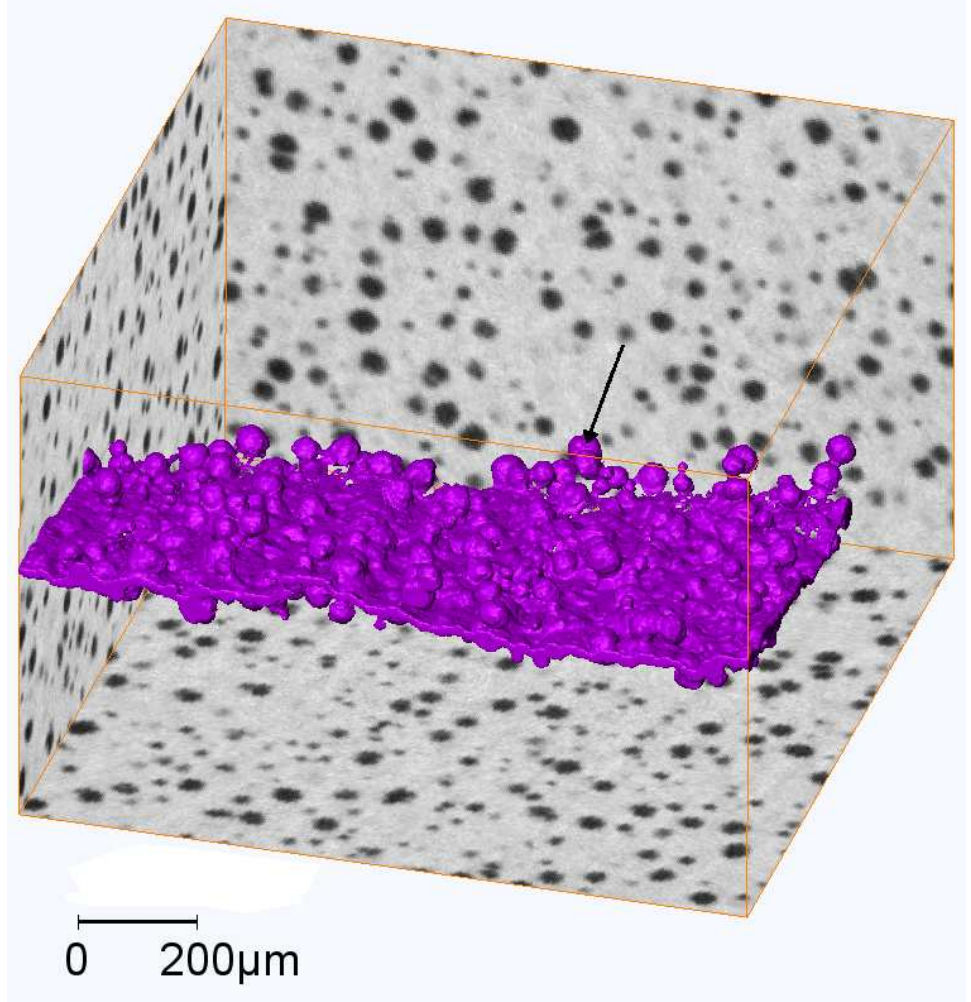

Fig. 4. 3D rendering of the crack surface after 45 kcycles obtained by classical thresholding of the tomographic image. Note that the nodules intersecting the crack surface are also imaged (arrow).

$186 \mathrm{~N}$, after 45,000 cycles. The crack appears to be slightly dissymmetric. The initial lengths on both sides of the specimen were measured with an optical microscope before the tomography experiment. It was equal to $580 \mu \mathrm{m}$ on the left-hand side of the specimen represented in Figure 5, and $796 \mu \mathrm{m}$ on the right-hand side. Due to tunneling effects, the pre-cracked larger specimen had a larger crack length in the center than at its edges. Thus, the smaller crack length in the tomography specimen machined from the larger one corresponds to the former free surface while the longer crack length corresponds to the former center.

The 3D rendering of the crack surface obtained from the error map strongly de- 


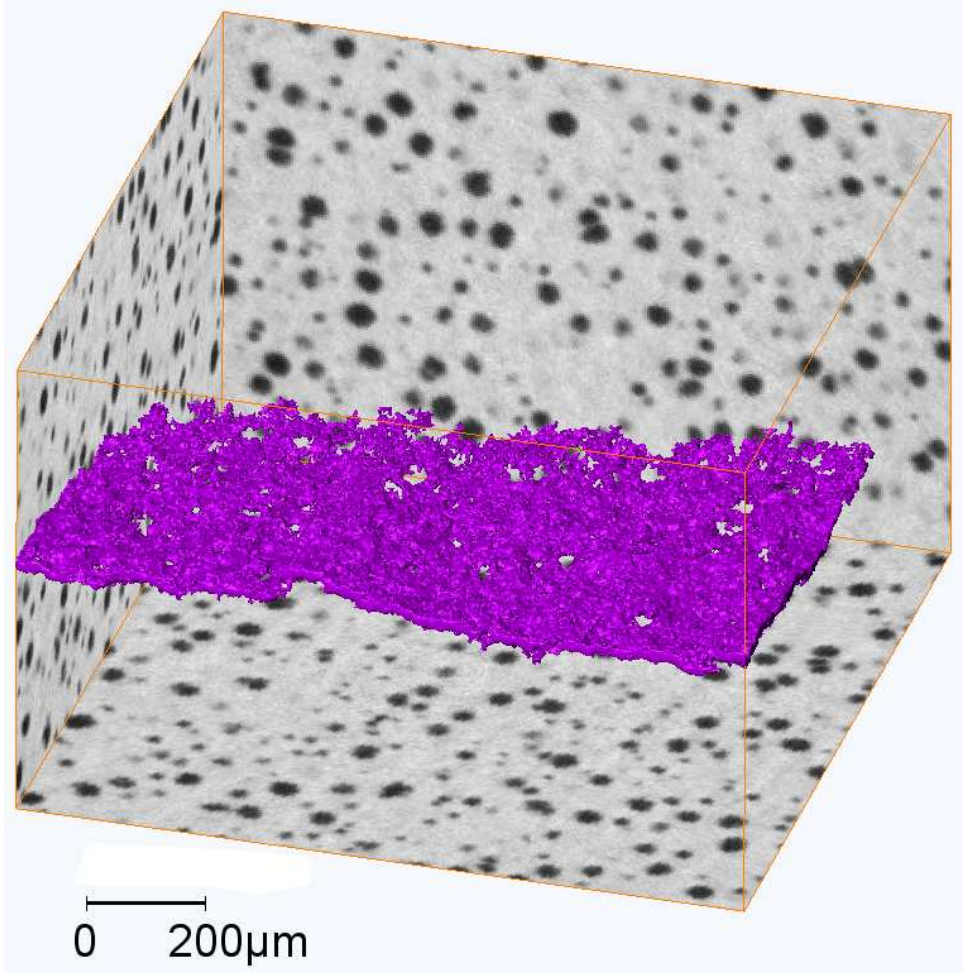

Fig. 5. 3D rendering of the crack surface after 45,000 cycles obtained by thresholding the correlation residual field.

pends on the threshold value used for the region growing algorithm. A threshold value that is too small may result in noise embedded in the thresholded crack while a value that is too large may underestimate the crack position, particularly close to the crack tip where the thickness of the 3D object to threshold is the smallest. Consequently, the crack tip was also measured directly from the tomographic image in gray levels corrected for the displacements measured by DIC. The methodology uses ImageJ software [53]. At maximum load, the crack is viewed edge on using a color lockup table with improved contrast to ease crack tip detection. An ImageJ plugin allows for the retrieval of the voxel coordinates that are manually selected at the crack tip. Thus, the crack tip position is manually tracked in the thickness of the specimen and the re- 
sulting points compare well with the thresholded error map (Figure 6). The initial crack lengths measured optically also corroborate the crack tip position for the very first cycle as shown in Figure 6.

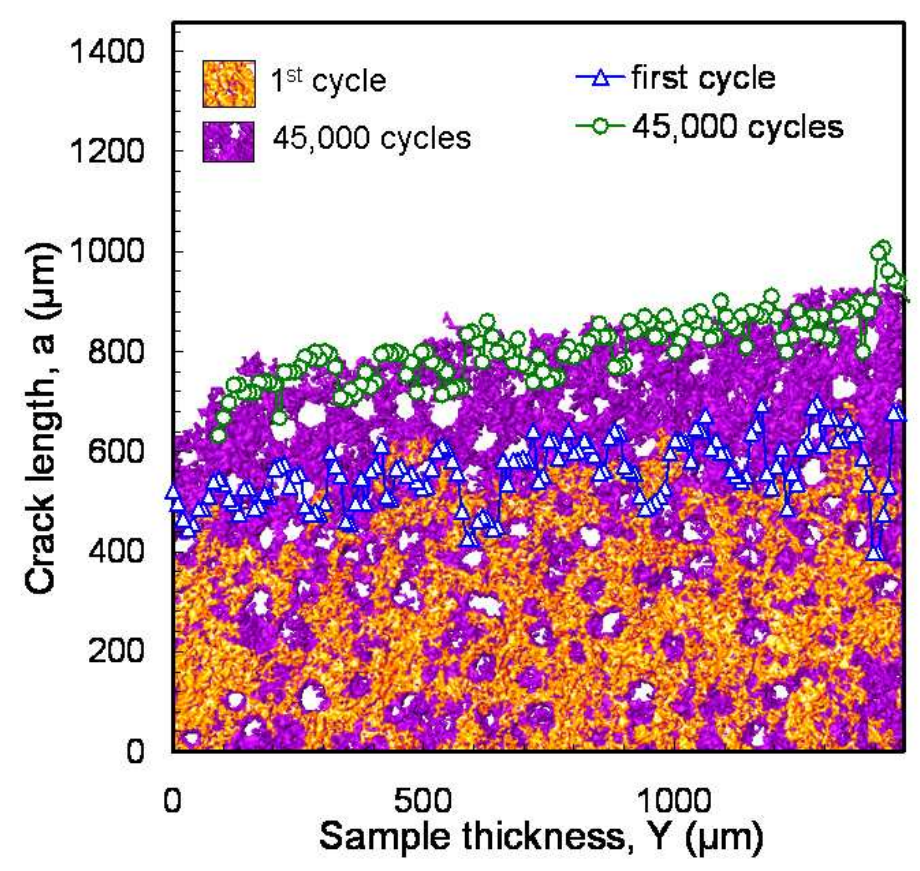

Fig. 6. Crack tip position: manual tracking (open symbols) compared with thresholded residual error fields.

\subsection{Multiscale crack detection algorithm}

The above described digital image correlation technique applied to the unloaded and maximum load image pair produces a residual error field that has high values concentrated on the crack surface, as shown in Figure 7. The latter shows a map of correlation residual in a through cut intersecting the crack surface. Only one cut is shown to illustrate this feature, but all cuts show similar characteristics.

Since the correlation residual is based on squared image differences after cor- 


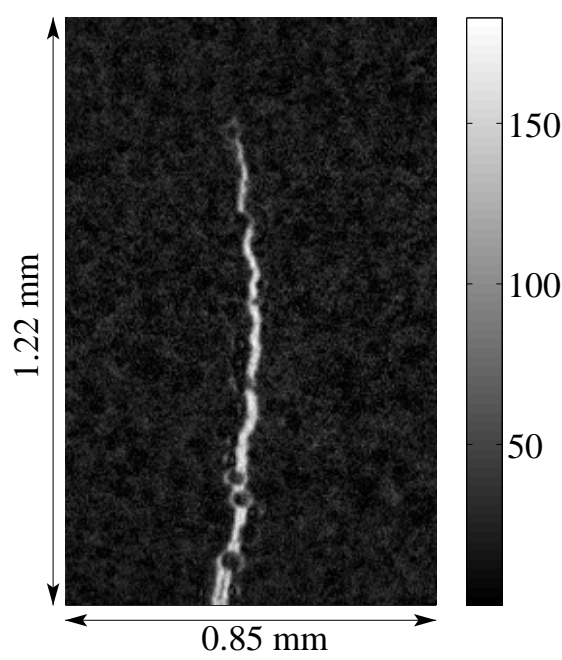

Fig. 7. Cut through the 3D correlation residual field normal to the crack front after 45,000 cycles. The crack is clearly visible close to the edge where it is widely open. The crack front is more difficult to appreciate accurately.

rection, both iron matrix and graphite nodules are essentially erased in the residual map. However, the crack itself remains clearly visible. It is therefore a strong motivation to further exploit this 3D field to adjust the local geometry of the cracked surface. However, it is also noteworthy that high error values remain in the field that may be due to imperfections in the $3 \mathrm{D}$ reconstruction, image noise, or deviation from brightness conservation.

The problem is addressed under the following form. The crack surface is represented as a surface $z(x, y)$ that contains no hole, nor overhangs. In this section only, let $\boldsymbol{x}$ denote the $(x, y)$ coordinates in a plane that is roughly parallel to the crack surface. One selects $z$ based on the maximization of

$$
\mathcal{S}=\max _{z(\boldsymbol{x})} \iint r^{2}(\boldsymbol{x}, z(\boldsymbol{x})) \mathrm{d} \boldsymbol{x}
$$

where $r$ denotes the residual density (see Equation (4)). Here again the problem is ill-posed, and requires a regularization, namely, $z$ is decomposed over a 
basis of (2D) finite element shape functions

$$
z(\boldsymbol{x})=\sum b_{i} \psi_{i}(\boldsymbol{x})
$$

The problem shares a number of similarities with the identification (or measurement) of the displacement field itself. Therefore, a similar strategy is followed. For each nodal value $\boldsymbol{x}_{i}$, a "driving force" $f_{i}$ to minimize the above energy is written as

$$
f_{i}=\iint \frac{\partial r^{2}}{\partial z}(\boldsymbol{x}, z(\boldsymbol{x})) \psi_{i}(\boldsymbol{x}) \mathrm{d} \boldsymbol{x}
$$

A relaxation algorithm based on the response to the above driving force is written. The difficulty is that the level at which $\mathcal{S}$ saturates is unknown and hence the previous algorithm cannot be followed down to this level. Instead, a "viscosity" $\eta$ is introduced so that

$$
\frac{\mathrm{d} b_{i}}{\mathrm{~d} t}=\eta f_{i}
$$

Again, the problem is unsafe because of the multiplicity of minima. Therefore the residual image is progressively coarsened up to the stage where only a single (but diffuse) crack remains. Then a finer image is used where the crack surface is initially positioned at the best location of the coarser image. This procedure is repeated down to the original residual field (without coarsening). The size of the mesh supporting the $\psi_{n}$ shape function is also a parameter that may be adjusted at different coarsening levels.

\subsection{Results}

In the present case, the residual image is coarsened 5 times, i.e., the coarsest voxel is $2^{4} \times 2^{4} \times 2^{4}$ initial voxels. For the coarsest level, the size of the 
elements supporting the $\psi_{n}$ shape functions is $2^{7} \times 2^{7} \times 2^{7}$ voxels and decreases down to $2^{2} \times 2^{2} \times 2^{2}$ voxels for the finest level.

The "viscosity" $\eta$ is adjusted in the following way. For the coarser scales, $\eta$ is evaluated so that the maximum increment of elevation at the first iteration on the current level does not exceed 0.1 grain (a grain being an aggregate of $2^{n} \times 2^{n} \times 2^{n}$ voxels). For the finest level, where a grain is a voxel, this maximum increment is set to 0.5 grain. Figure 8 shows the detected crack surfaces after convergence was reached at each scale. One observes the progressive resolution of local variation of the crack surface. From the coarsest to the finest scales, high frequencies are progressively introduced and resolved by the multi-grid discretization of the elevation and the coarsening of the residual map.

Figure 9 illustrates the convergence of the detection algorithm. A criterion is formulated in terms of an increment between two successive iterations of the norm of the elevation increment normalized by the norm of the elevation at the first iteration of the current scale. One observes a very fast convergence for the coarser scales. For the finer scales, the convergence rate decreases because a constant viscosity was adopted. Had the latter been adapted for each scale, a constant convergence rate could have been obtained.

Although the maximum of the objective function cannot be determined without knowing the conformation of the crack surface, one can easily derive an upper bound. Let us define $R^{+}$as the integral over the region of interest of the maximum value of the residual over each line normal to the crack surface. This value can be compared to the sum of the residuals over the detected crack at the current iteration, and thus an indirect indication of the convergence is obtained. Figure 10 shows such a distance from the current objective function 

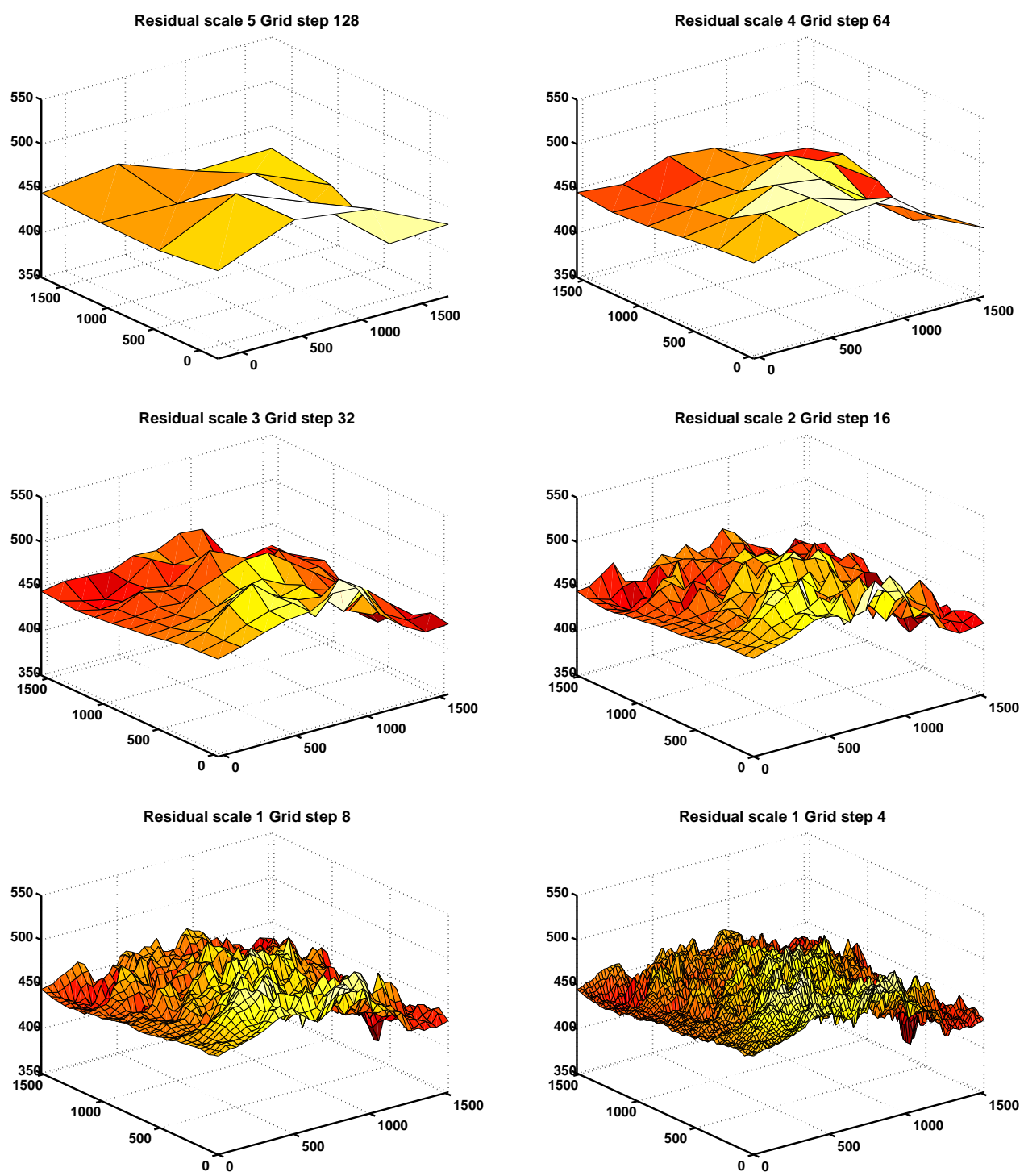

Fig. 8. Detected crack surfaces after convergence at each scale. Note that the roughness is enhanced in these graphs because of the $z$ scale which is dilated as compared to the in-plane axes. Axes are labeled in micrometers.

to this upper bound as a function of the iteration number. For the first iterations of each scale, a fast decrease of the normalized gap between $\mathcal{R}^{+}$and the actual residual sum is obtained. It is also observed that each scale brings an improvement and a final value of $7.75 \%$ is obtained (to be compared with the initial $9 \%$ obtained when a straight plane is considered). 


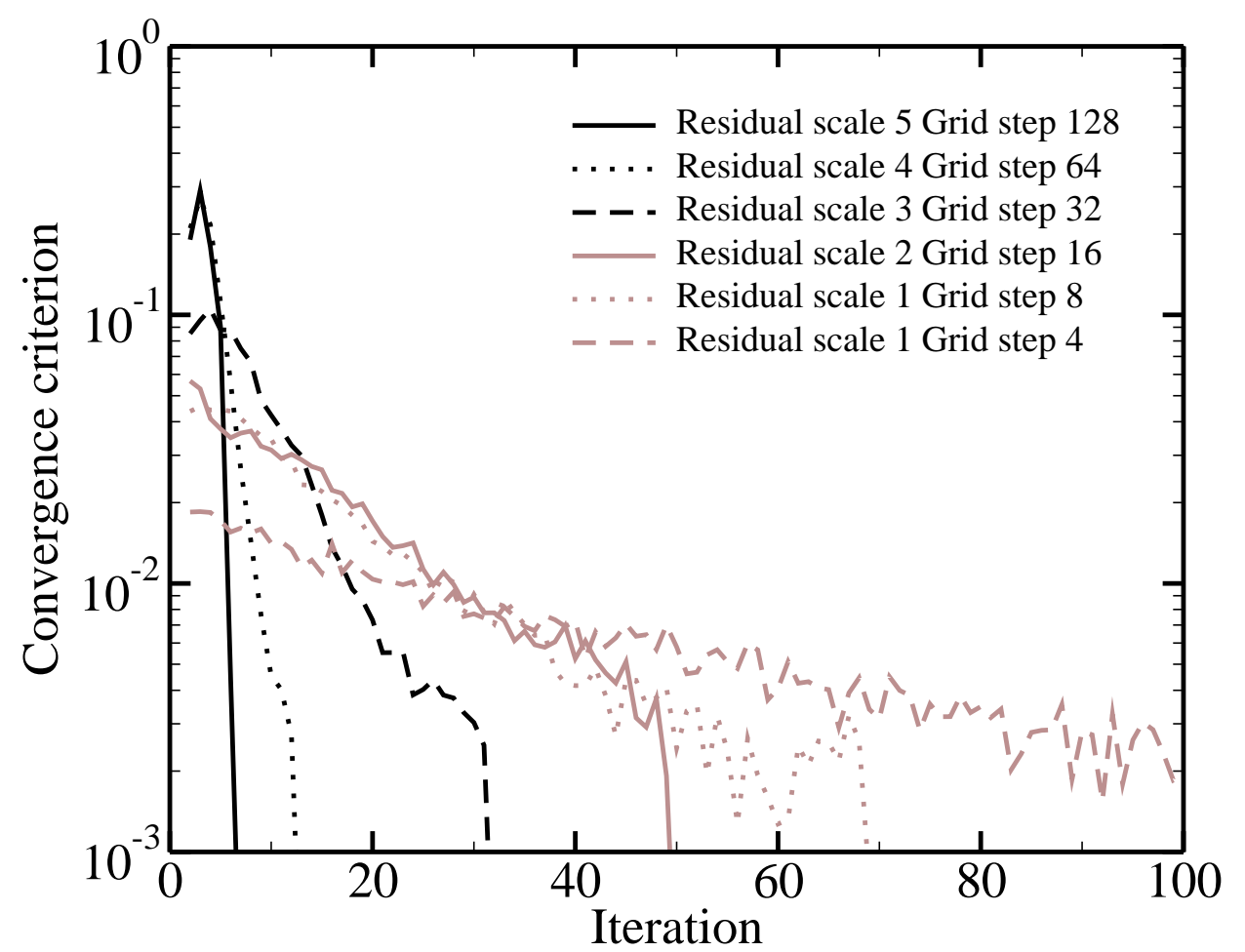

Fiq. 9. Convergence of the crack detection alonrithm.

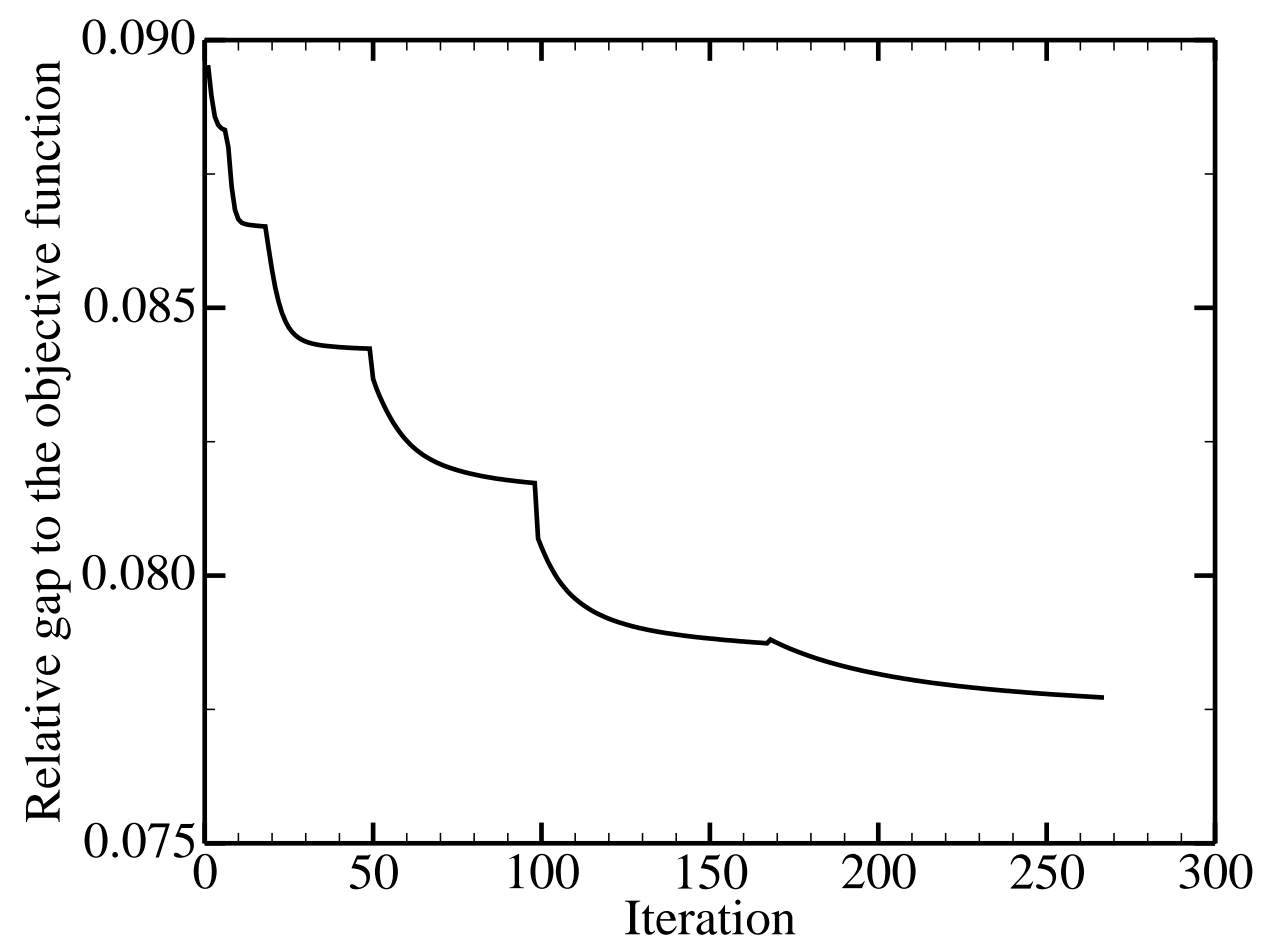

Fig. 10. Normalized gap between the sum of the residual on the detected crack and the upper bound to the objective function. 


\subsection{Comparison between different approaches}

Both crack detection methods based on the correlation residual map obtained from C8-DIC give a good estimate of the cracked surface visible in the tomographic image (Figure 11). However, a difficulty lies in positioning the crack tip. This information may be retrieved directly from tomographic image analysis via manual tracking of the crack tip through the specimen thickness; the good correlation that was obtained between the thresholded DIC residuals and the crack tip position (Figure 6) confirms the validity of the crack detection procedure based on the residual map. However, the crack surface identified via DIC residuals has a rather irregular surface and it contains holes where the nodules are embedded in the crack. The multi-scale crack detection algorithm of sub-Section 4.2 gives a smoother crack surface with the required topology. The crack surface so-obtained coincides with the one obtained by thresholding the DIC residuals except for high frequency "roughness" (with a period less than the smallest grid step of the detection algorithm). Note that the multi-scale crack detection algorithm provides a virtual crack surface over the non-cracked part of the specimen. The front line from the thresholded DIC residuals is then placed on this surface.

\section{3D eXtended Digital Image Correlation}

\subsection{Enrichment strategy}

If a discontinuity in the displacement is sought, the displacement interpolation is enriched exploiting Partition of Unity properties [51] of finite element shape 

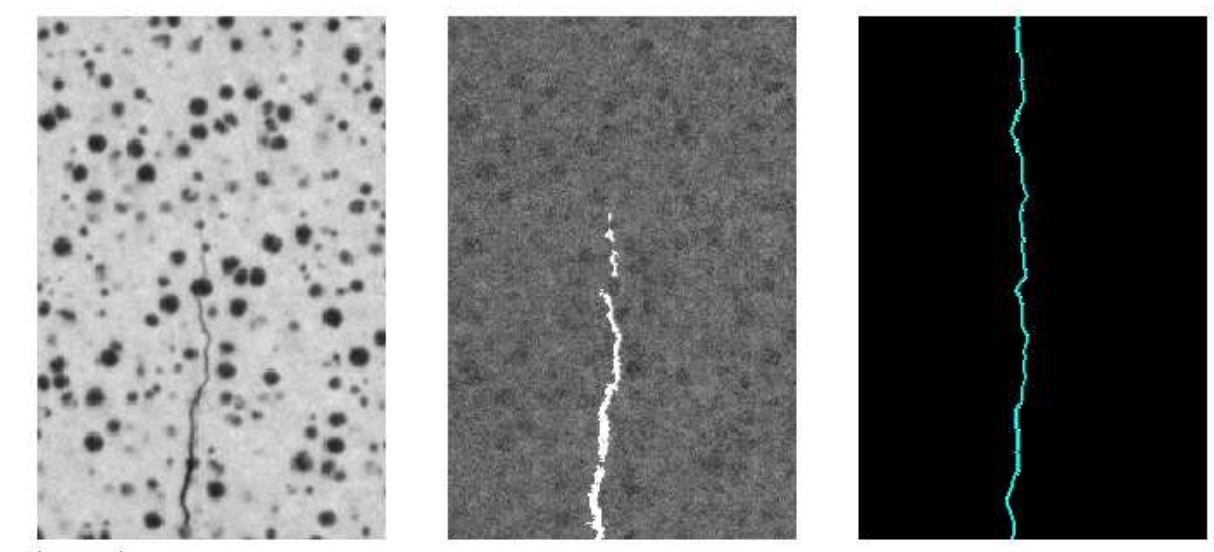

$200 \mu \mathrm{m}$

Fig. 11. Comparison after 45,000 cycles of a section through the tomographic image, the thresholded residual and the crack obtained with the multi-scale detection algorithm.

functions $N_{n}$

$$
\sum_{n \in \mathcal{N}} N_{n}(\boldsymbol{x})=1
$$

The enriched approximation of the displacement field, following a strategy initially proposed by Moës et al. [29], reads

$$
\boldsymbol{u}(\boldsymbol{x})=\sum_{j=1,3} \sum_{n \in \mathcal{N}} a_{n j} N_{n}(\boldsymbol{x}) \mathbf{X}_{j}+\sum_{j=1,3} \sum_{n \in \mathcal{N}_{c u t}} d_{n j} N_{n}(\boldsymbol{x}) H_{n}(\boldsymbol{x}) \mathbf{X}_{j}
$$

where $\left(a_{n j}\right)_{n \in \mathcal{N}}$ are degrees of freedom associated with standard (i.e., continuous) shape functions in each direction $\mathbf{X}_{1}, \mathbf{X}_{2}$ and $\mathbf{X}_{3}$ of the image co-ordinate system, $\mathcal{N}_{\text {cut }}$ the set of nodes that hold additional degrees of freedom $d_{n j}$ associated with the enrichment function $H_{n}(\boldsymbol{x})$ defined as [30]

$$
H_{n}(\boldsymbol{x})=\mathcal{H}(\boldsymbol{x})-\mathcal{H}\left(\boldsymbol{x}_{n}\right)
$$

where $\mathcal{H}$ is the Heaviside step function whose value is 0 below the crack surface and 1 above (Figure 12), and $\boldsymbol{x}_{n}$ gives the position of node $n$.

For three-dimensional problems, the pioneering work of Sukumar et al. [31] first used XFEM approaches to 3D cracks, and Moës et al. [32] promoted the 


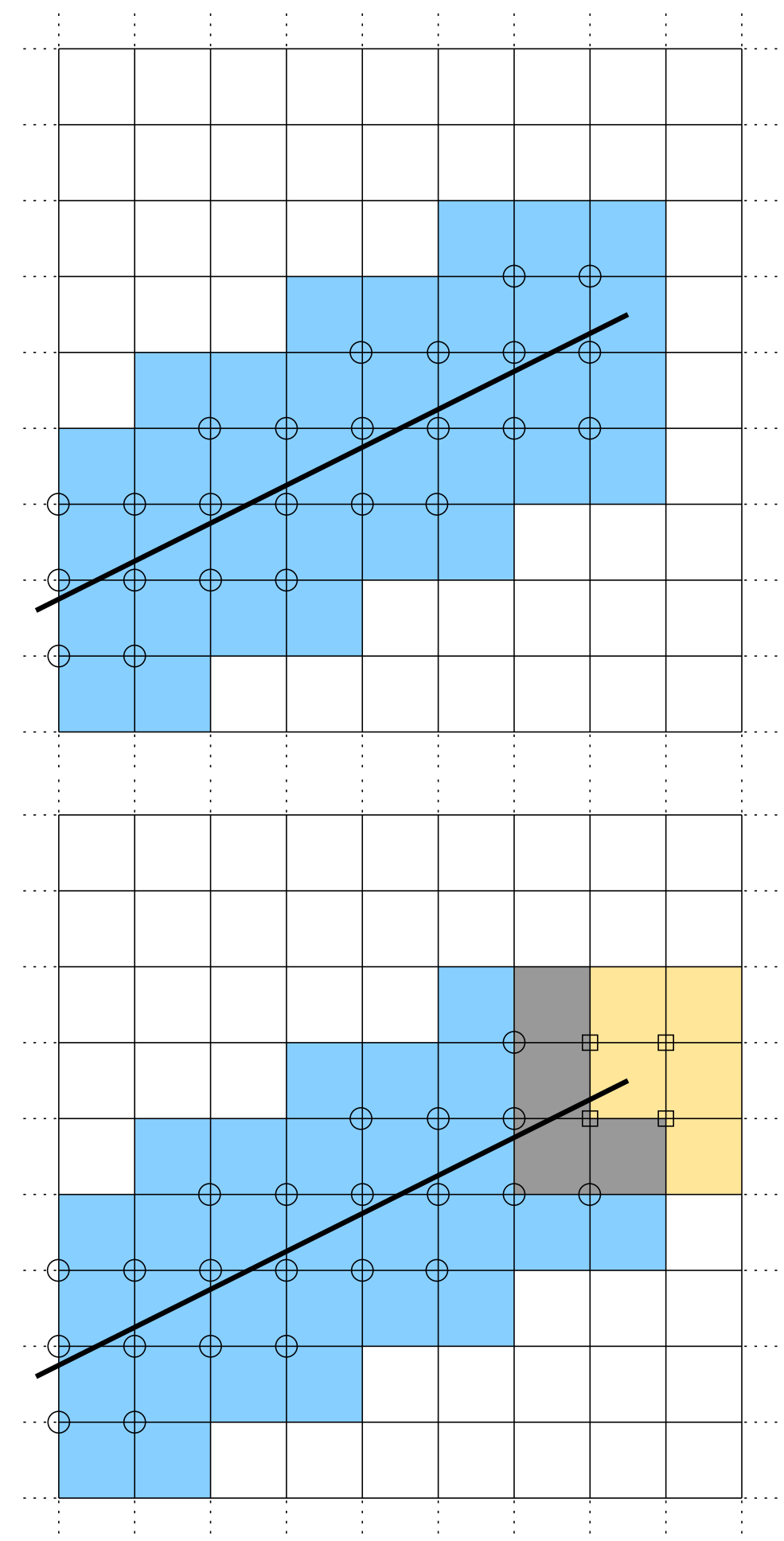

Fig. 12. Enrichment strategies for extended digital image correlation (top) and extended finite element method (bottom). Circles denote nodes with a discontinuous enrichment and squares those with asymptotic enrichment functions [32]. Filled elements define the support of the enrichments. 
use of the description the crack surface using two level set fields. These level sets are the signed distance to the crack surface and the signed distance to the crack front under orthogonality constraints. The identification of the crack geometry was described in the previous section. In the present case, the level set fields are discretized on a finite difference grid independent of the finite element mesh used for the displacement estimation. For the DIC technique, the finite difference grid is the 3D voxel grid of the reference image. It allows for a better computational efficiency when using propagation, re-orthogonalization or re-initialization algorithms to describe crack propagation. In the following, the enrichment procedure is applied to a $\mathrm{C} 8$ approach (C8-DIC). It is therefore referred to as $\mathrm{X}-\mathrm{C} 8$.

\subsection{A priori performance}

An important feature is the choice of the element size. To decide for this characteristic, a methodology has been set in place, namely, a uniform translation is artificially prescribed on the reference image to compute a fictitious "deformed" image. To reveal the most severe effects of sub-voxel interpolation, a series of constant sub-voxel displacement in each direction $x, y$ and $z$ is prescribed. A blind analysis of the displacement is then run, and the resulting displacement field is compared to the prescribed value. The mean (over all those displacements) of the mean difference $\eta_{u}$ (i.e., systematic error) and the corresponding standard deviation $\sigma_{u}$ (i.e., displacement uncertainty) of the nodal displacements

$$
\eta_{u}=\left\langle\mathbf{u}-\mathbf{u}_{p}\right\rangle \quad, \quad \sigma_{u}=\left\langle\left\|\mathbf{u}-\mathbf{u}_{p}\right\|^{2}\right\rangle^{1 / 2}
$$


give a good a priori evaluation of the maximum performance of the analysis, where $\mathbf{u}_{p}$ is the prescribed displacement, and $\langle\ldots\rangle$ denotes the average value over the nodes of the entire region of interest. The mean (systematic) error $\eta_{u}$ and standard uncertainty $\sigma_{u}$ are evaluated for different element sizes varying from 8 to 64 voxels.

When a rigid body translation is prescribed, the $\mathrm{X}-\mathrm{C} 8$ discretization gives the same results as a C8 scheme (Figure 13). It is generally observed that both quantities, $\eta_{u}$ and $\sigma_{u}$ decrease as a power-law of the element size. Such a behavior is observed for the uncertainty, but the systematic error seems to reach a minimum and increase again for larger element sizes. This effect may be due to the small number of elements needed to pave the region of interest, and where boundary elements may bias the overall behavior in a significant fashion. Larger elements mean less degrees of freedom for the same amount of data available (region of interest) and thus a better conditioning of the problem and a more robust estimation of the displacement field are achieved. A mean systematic error as low as $10^{-3}$ voxel associated with a mean standard deviation of about $10^{-2}$ voxel are obtained. It is thus concluded that the enrichment does not affect the determination of regular degrees of freedom.

Second, a discontinuous translation is prescribed along a discontinuity plane that separates the region of interest into two domains of equal size. Focusing on the regular degrees of freedom only (Figure 14), similar trends as in the previous case are obtained for an X-C8 approach, whereas a C8 measurement shows a "non-converging" behavior. The prescribed displacement, which is discontinuous, cannot be approximated exactly by a C8 discretization, an interpolation error is obtained and thus decreases the quality of the displacement estimate. When $\mathrm{X}-\mathrm{C} 8$ is used, the same type of results are shown for the 

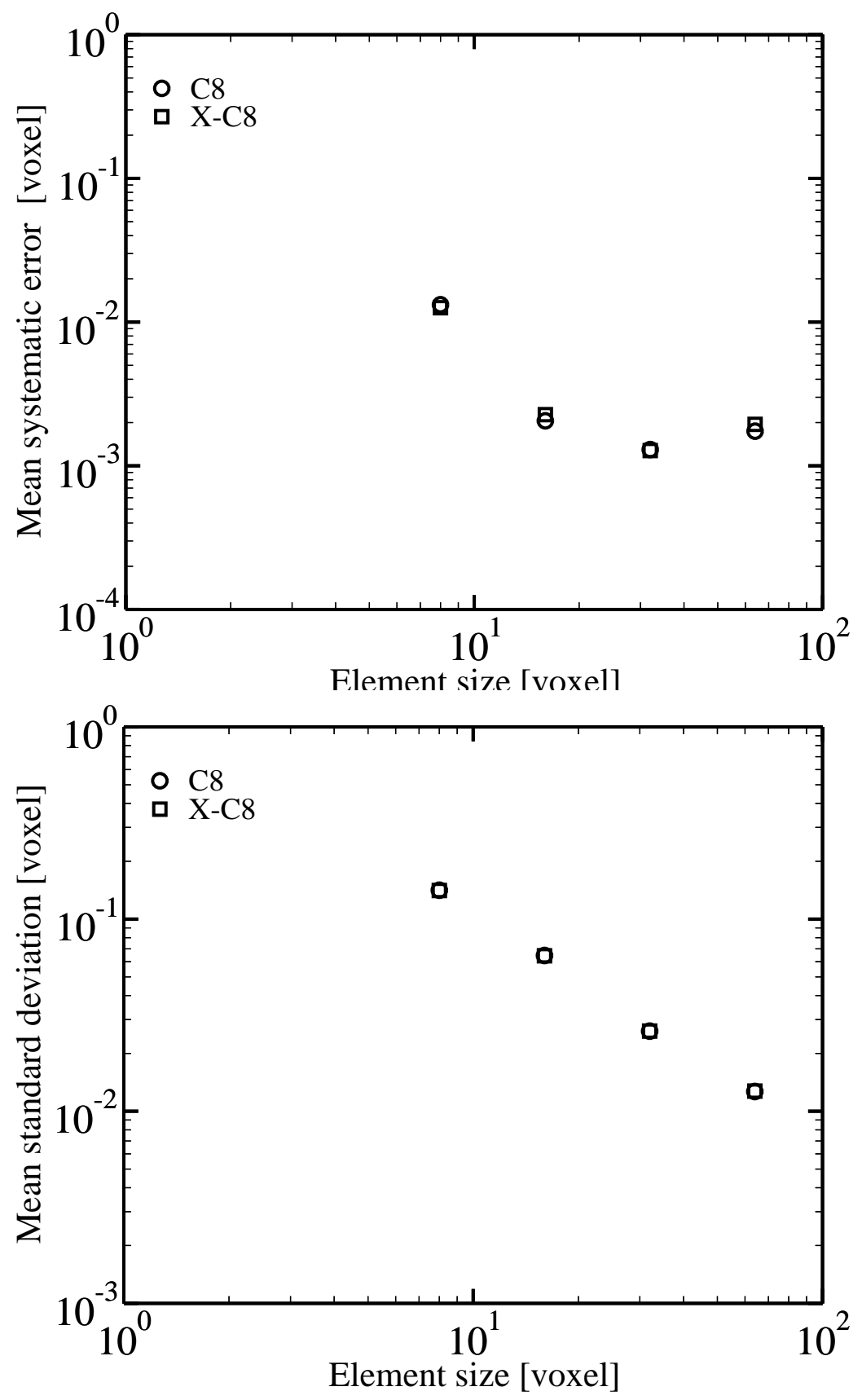

Fig. 13. Mean systematic error and standard deviation for a prescribed uniform displacement ranging between 0 and 1 voxel obtained for $\mathrm{C} 8$ and $\mathrm{X}-\mathrm{C} 8$ approaches. enhanced discontinuous degrees of freedom in Figure 15. Globally, a significant decrease in systematic error and uncertainty is observed when using the enriched description. This leads to the choice of elements of size 32 voxels for which $10^{-3}$-voxel mean systematic error, and $2 \times 10^{-2}$-voxel mean standard 

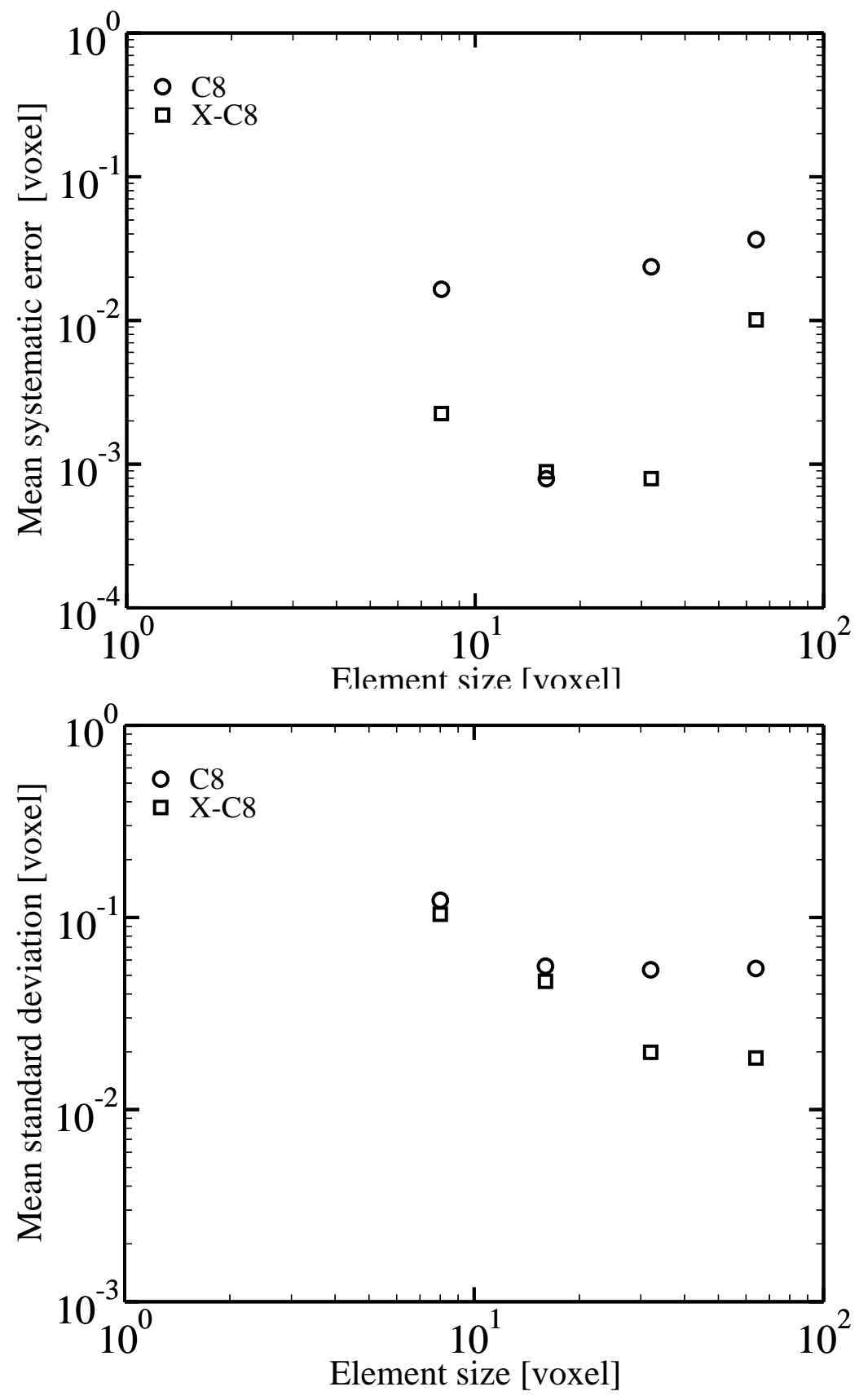

Fig. 14. Mean systematic error and standard deviation for a prescribed discontinuous displacement ranging between 0 and 1 voxel obtained for $\mathrm{C} 8$ and $\mathrm{X}-\mathrm{C} 8$ approaches.

deviation are estimated.

It is concluded that the enrichment of the interpolation basis not only allows for an estimation of a displacement discontinuity but also improves the de- 

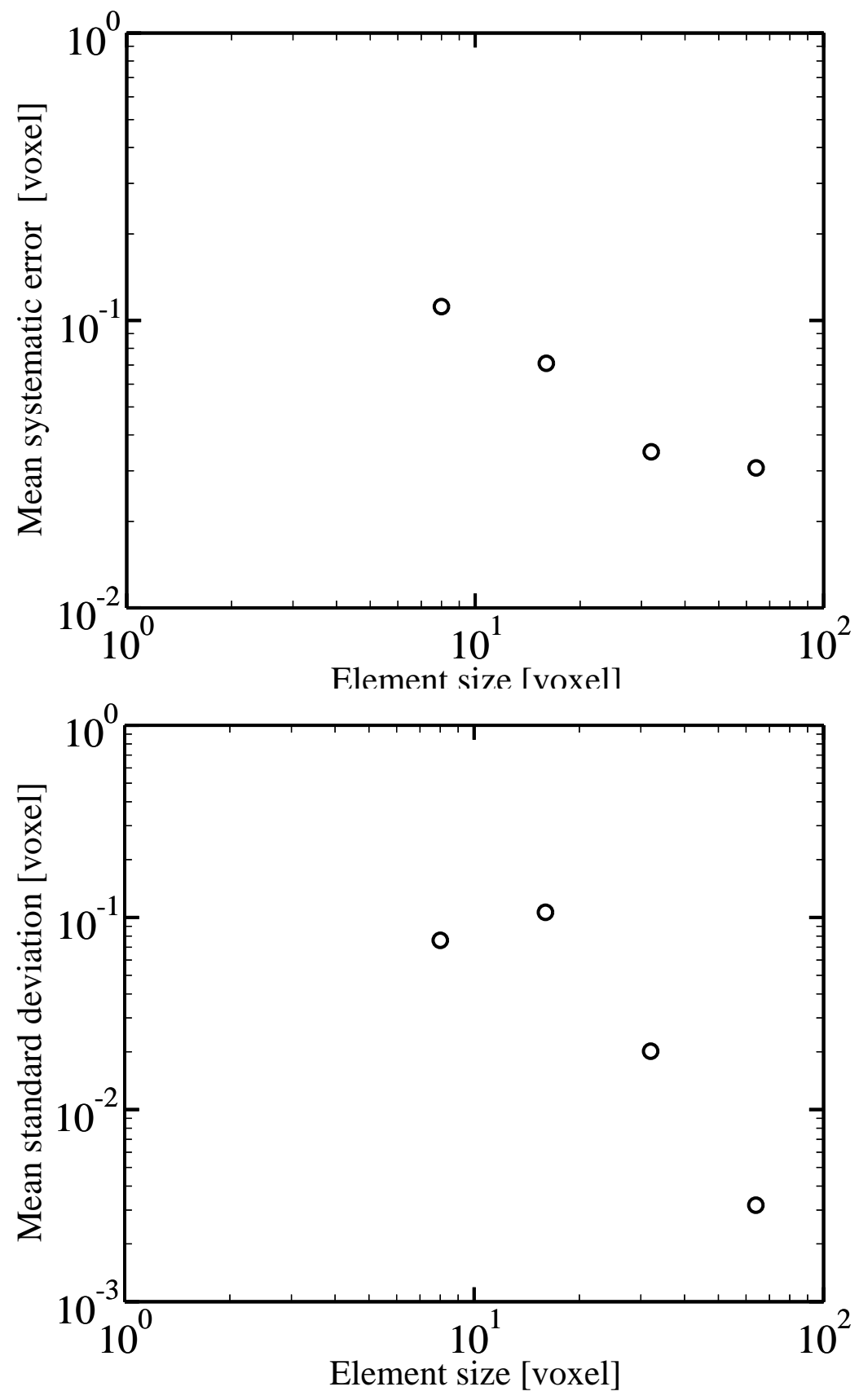

Fig. 15. Mean systematic error and standard deviation for the displacement jump when a discontinuous displacement ranging between 0 and 1 voxel is prescribed obtained and an X-C8 approach is used.

termination of degrees of freedom associated with the regular basis when a discontinuous displacement is searched for. Note that in the latter analysis, the support of the discontinuity that cuts the elements at the middle of their 
edges is an idealized case.

\subsection{Results}

Having validated the $\mathrm{X}-\mathrm{C} 8$ procedure, it is now used to analyze the experiment described in Section 2. The displacement field obtained with 32-voxel elements with discontinuous enrichment is shown in Figure 16 for the maximum load level after 45,000 cycles. The region of interest is $288 \times 288 \times 288$ voxels centered in the reference image. The component $U$ of the displacement field is along the loading axis (orthogonal to the crack surface). A discontinuity is captured for this component but also for $V$ corresponding to an out-plane sliding of the crack face. The third component $W$ has no enriched degrees of freedom activated (up to the measurement uncertainty). In the standard C8-DIC, small elements (16 voxels) are needed to obtain a satisfactory description of the displacement field near the crack surface (see Figure 3). As the enrichment of the $\mathrm{X}-\mathrm{C} 8$ method enables one to capture the discontinuity of the displacement field, larger elements are used (32 voxels). Thus, it leads both to lower noise levels of the displacement field in the bulk and to an appropriate description of the displacement field around the crack.

Figure 17 describes the change of the mode $I$ crack opening displacement (i.e., jump of the $U$ component) on the crack surface for different load levels. One observes that the crack progressively opens with a predominant opening around the edge along which it is longer. The maximum opening is about 1.25 voxel (or $6.3 \mu \mathrm{m})$. 

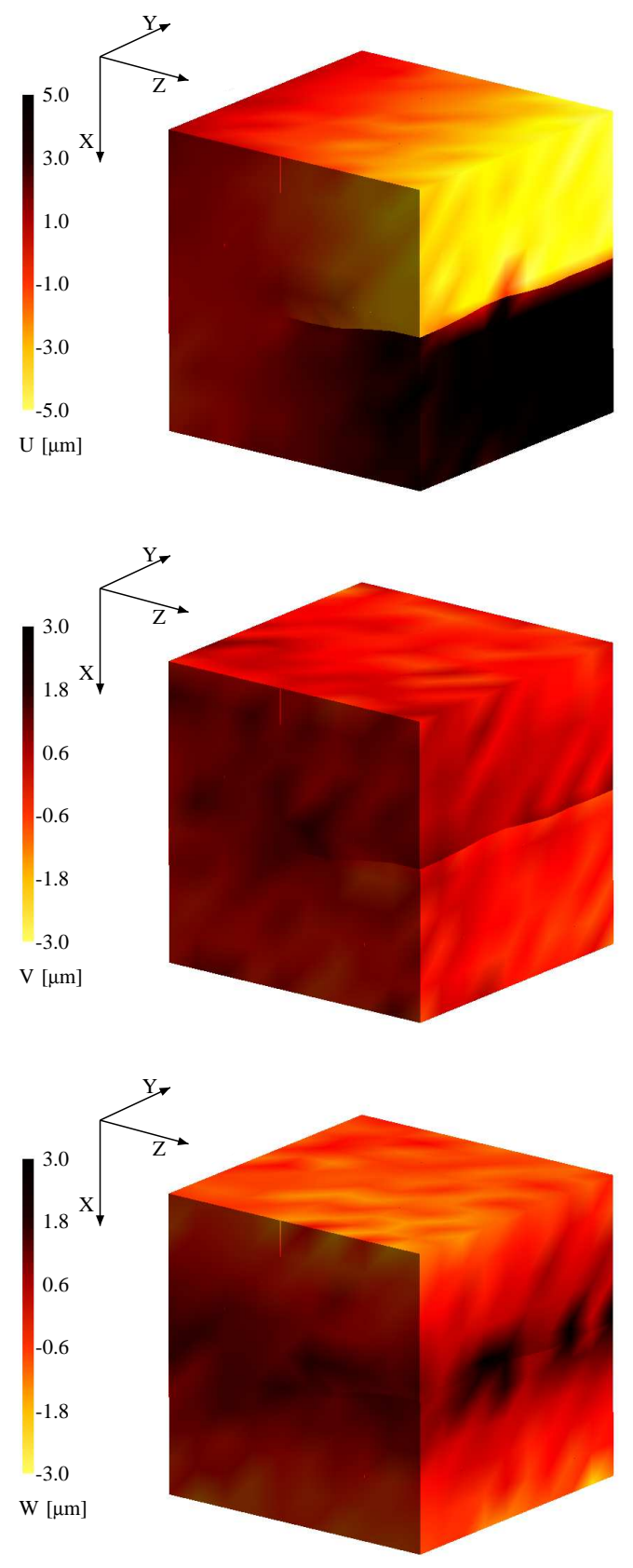

Fig. 16. The three components of the displacement field (in micrometers) for the maximum load level after 45,000 cycles with an X-C8 approach. The mean rigid body motion was extracted from those fields. The volume shown in this figure is a cube whose edge is $1.46-\mathrm{mm}$ (or 288-voxel) long. 

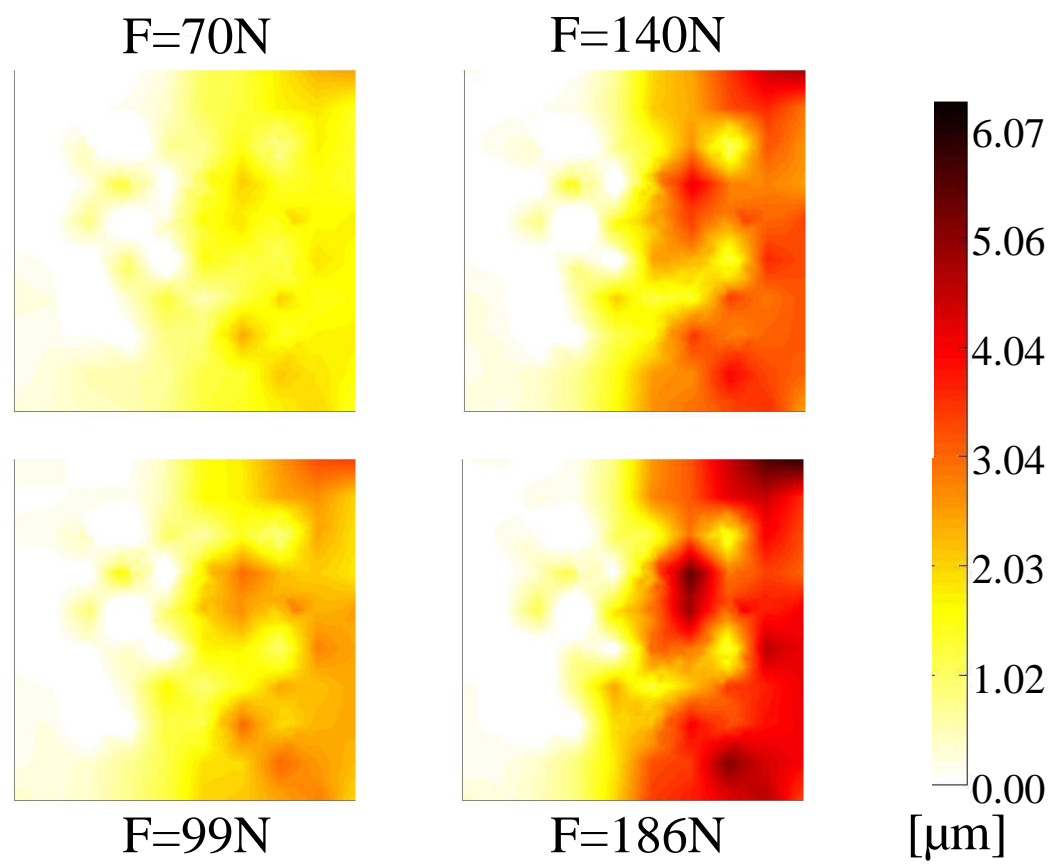

Fig. 17. Change of the mode I crack opening displacement on the crack surface for different loads after 45,000 cycles with an X-C8 approach.

\section{Elastic computation on the same geometry and boundary con- ditions}

The previous sections have detailed the X-DIC strategy, and the operational way of defining the crack surface. With those elements at hand, it is natural to perform an elastic computation based on exactly the same geometry. This will allow for a detailed comparison between the measured and computed displacement fields. Let us however stress an important point, namely, the digital image correlation data are corrupted by measurement uncertainties, yet on average they represent the actual material with its full complexity (microstructure, confined plasticity, rough crack surface). Conversely, the computed displacement field is almost deprived of any noise, but, for instance, it ignores the effect of crack tip plasticity. Because of confinement, this may not be essential. However, the actual geometry of the crack front and the "equiv- 
alent" one that will produce, in the far field, the same elastic solution may not coincide. This effect is ignored and the coincidence of the two fronts is assumed.

\subsection{Computation hypotheses}

In this respect, a robust interfacing tool is developed to introduce the "real" crack shape in the numerical model. For that purpose, a finite difference based level set description is obtained from 3D pictures, or digital volume correlation $[9,37,54-56]$. The advantages of a level set modeling on an external finite difference mesh is summarized as follows. The finite difference mesh is adapted to the area of interest, and the mesh size adapted to the experimental crack shape $[9,37]$. Furthermore, level sets are very efficient to model non-planar surfaces even if they evolve with time [54-56], or exhibit variable topological properties. Last, it was shown that level sets are well suited to X-FEM in order to introduce automatically specific enrichments according to the crack and the front during the possible propagation of the crack, and to improve the accuracy of Stress Intensity Factor (SIF) calculations with adapted virtual crack extension fields [55].

From this initialization step, it is then possible to couple the "real" crack level set model with the eXtended Finite Element Method. Specific discontinuous and asymptotic enrichments are automatically introduced in the finite element mesh to capture the "mechanical" discontinuity and the asymptotic behavior close to the crack front [55]. These enrichments avoid the mesh dependence of the initial crack shape and its possible propagation, with a sufficient accuracy [37]. However, the finite element size is an important parameter in the 
area of interest (close to the crack front). Some previous studies have shown that a local multi-grid strategy may be used to capture the possible scale effects close to the crack front in a very efficient way. In this respect, a local fine grid is used in a narrow band close to the "real" crack, and a coarse grid is used to mesh the structure and the boundary effects.

\subsection{Local multi-grid X-FEM strategy}

As explained in the previous section, the level set can be automatically initialized according to experimental data. However, the different scales involved in fracture mechanics problems may differ by several orders of magnitude and an arbitrary finite element mesh of a given structure is usually not designed to account for a crack [57]. Even with X-FEM, enrichments are useless if the crack size is smaller than 3-4 times the element size of the given initial mesh. To overcome this drawback, a local multi-grid eXtended Finite Element Method is proposed to adapt the mesh in the area of interest and the possible crack propagation.

One defines a local "fine" grid in a narrow band close to the crack, a "coarse" grid linked to the global structure, and possible intermediate grids between the locally fine and the globally coarse ones. The efficiency of multi-grid algorithms lies in the fact that only a small amount of work is required to solve the expensive fine problem $[58,59]$. The major part of work is dedicated to solving the coarse problem, which is much less expensive. Using a sequence of grids increases the multi-grid efficiency. Furthermore, a ratio of 2 between two successive hierarchical grids seems to be optimal [37]. Instead of being exactly solved, the coarse problem is approximated by performing recursive calls to 
the multi-grid procedure, using a still coarser discretization. In Table 1, one summarizes the multi-grid algorithm that consists in iterations between the coarse (AB) and fine (B) grids, where A concerns the non-overlapping region, and $\mathrm{B}$ the overlapping part between the coarse and fine meshes.

Table 1

The two level multi-grid algorithm.

(1) initialization

$$
\begin{aligned}
k & =0 \\
\overline{\mathbf{U}}^{B} 0 & =0 \\
\mathbf{U}^{B} 0 & =0
\end{aligned}
$$

(2) compute the solution of the coarse problem

$$
\overline{\mathbf{K}}^{A B} \overline{\mathbf{U}}^{k+1}=\overline{\mathbf{F}}-\left[\mathbf{P}^{t} \mathbf{K}^{B} \mathbf{U}^{B^{k}}\right]+\left[\overline{\mathbf{K}}^{B} \overline{\mathbf{U}}^{B^{k}}\right]
$$

(3) correction

$$
\begin{aligned}
& \Delta \overline{\mathbf{U}}^{B^{k+1}}=\overline{\mathbf{U}}^{B^{k+1}}-\overline{\mathbf{U}}^{B^{k}} \\
& \mathbf{U}^{B^{k+1 / 2}}=\mathbf{U}^{B^{k}}+\mathbf{P} \Delta \overline{\mathbf{U}}^{B^{k+1}}
\end{aligned}
$$

(4) relaxation step (fine problem): small number of PCG iterations $(\nu<5)$ on

$$
\mathbf{K}^{B} \mathbf{U}^{B^{k+1}}=\mathbf{F}_{e x t}^{B} \quad \text { with }\left.\mathbf{U}^{B^{k+1}}\right|_{\Gamma}=\left.\mathbf{P} \overline{\mathbf{U}}^{B^{k+1}}\right|_{\Gamma}
$$

(5) convergence test

$$
\left\{\begin{array}{l}
\text { if } \frac{\left\|\mathbf{U}^{B^{k+1}}-\mathbf{U}^{B^{k+1 / 2}}\right\|}{\left\|\mathbf{U}^{B^{k+1}}\right\|}<\varepsilon \\
\text { else } \\
\qquad: k \leftarrow k+1 \text { and go back to } 2
\end{array}\right.
$$


The notation $\left[\mathbf{X}^{B}\right]$ means that a vector related to the overlapping coarse mesh $\overline{\mathcal{M}}^{B}$ is extended to the whole coarse mesh $\overline{\mathcal{M}}$ by adding 0 values corresponding to nodes belonging to $\overline{\mathcal{M}}^{A}$ only.

This algorithm exploits the property of iterative solvers such as preconditioned conjugate gradients (PCG) to capture efficiently the high frequency components of the solution (according to the discretization size) during the smoothing or relaxation step on the fine grid, and the ability of the coarse grid to capture the low frequency components of the solution [60]. However, it also needs one to define prolongation $\mathbf{P}$ and restriction $\mathbf{R}$ operators between the different grids. In a previous paper [37], it was shown that the following property can be preserved even with enrichments

$$
\mathbf{R}=\mathbf{P}^{t}
$$

where the superscript $t$ denotes matrix transposition. Furthermore, the possible high frequency errors introduced locally (i.e., close to the crack front) are immediately captured by the relaxation steps. Another technical aspect about the local multi-grid X-FEM algorithm concerns the introduction of enrichments at each level of the grid. It was also shown [37] that an optimal rate of convergence is achieved when the characteristic element size of the starting enrichment level is of the order of magnitude of the crack size. This property will automatically be used from now on. The last point to be addressed is the definition of the level sets in a separate finite difference mesh. Usually, the crack surface and the front level sets are defined on the mesh of the structure. In the case of a local multi-grid approach, this would imply one to define independently the level sets on the different grids and to update all these different scalar fields with their own discretizations. The use of a single structured independent mesh only devoted to the representation of the crack 
morphology avoids such a drawback (Figure 18).

Last, the following enrichments are introduced according to the level sets [32] in the local basis along the crack front (Figure 12)

$$
\begin{array}{r}
\boldsymbol{u}(\boldsymbol{x})=\sum_{j=1,3} \sum_{n \in \mathcal{N}} a_{n j} N_{n}(\boldsymbol{x}) \mathbf{X}_{j}+\sum_{j=1,3} \sum_{n \in \mathcal{N}_{p c u t}} d_{n j} N_{n}(\boldsymbol{x}) H_{n}(\boldsymbol{x}) \mathbf{X}_{j} \\
+\sum_{j=1,3} \sum_{n \in \mathcal{N}_{t i p}} \sum_{e=1,4} s_{e j} N_{n}(\boldsymbol{x}) F_{e}(\boldsymbol{x}) \mathbf{X}_{j}
\end{array}
$$

with

$$
\begin{array}{ll}
F_{1}(r, \theta)=\sqrt{r} \cos \frac{\theta}{2} & F_{3}(r, \theta)=\sqrt{r} \cos \frac{\theta}{2} \sin \theta \\
F_{2}(r, \theta)=\sqrt{r} \sin \frac{\theta}{2} & F_{4}(r, \theta)=\sqrt{r} \sin \frac{\theta}{2} \sin \theta
\end{array}
$$

and $\mathcal{N}_{\text {pcut }}$ the set of nodes that hold discontinuous enrichments and $\mathcal{N}_{\text {tip }}$ those that hold singular enrichments. It is worth noting that the grids are enriched only if the size of the elements is of the order of the crack length in order to optimize the convergence rate of the multi-grid algorithm [37].

\subsection{Results}

In this paragraph, one considers the $3 \mathrm{D}$ geometry of the specimen analyzed with digital image correlation (Section 4). In this first approach, one defines a $3 \mathrm{D}$ beam with a length equal to $7.0 \mathrm{~mm}$ (cross section $1.6 \times 1.6 \mathrm{~mm}^{2}$ ). This allows one to avoid the boundary effects due to the prescribed loads. One assumes the material to be homogeneous, isotropic, with the properties given in Section 2. One also considers a prescribed load equal to $186 \mathrm{~N}$ (a corresponding uniform pressure is defined at the ends of the beam). The crack shape modeling consists in defining an external structured mesh in the area of interest. From digital image correlation, one obtains the crack shape, and the corresponding level sets for the crack surface and front on the structured 


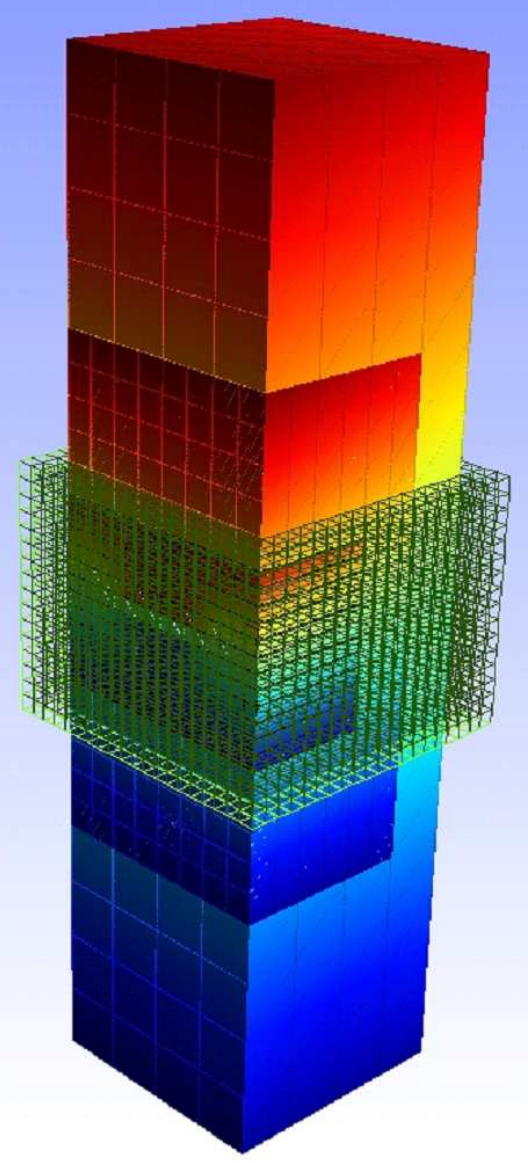

Fig. 18. Multi-grid mesh (3 levels) and external level set grid.

mesh. Furthermore, the finite element mesh of the beam is defined according to the area of interest containing the crack. If needed, different levels of grids are considered from a global (coarse) mesh of structure (400- $\mu$ m elements) to a local (refined) mesh close to the crack (100- $\mu$ m elements). The external level set mesh size is equal to $100 \mu \mathrm{m}$, having here the same refinement as the local finest X-FEM grid.

In Figure 19, the crack surface and front level sets are shown according to the analysis of Section 4. The crack surface is obtained through the multi-scale crack detection algorithm (Figure 8) and the front is located on the surface by manual tracking (Figure 6). 


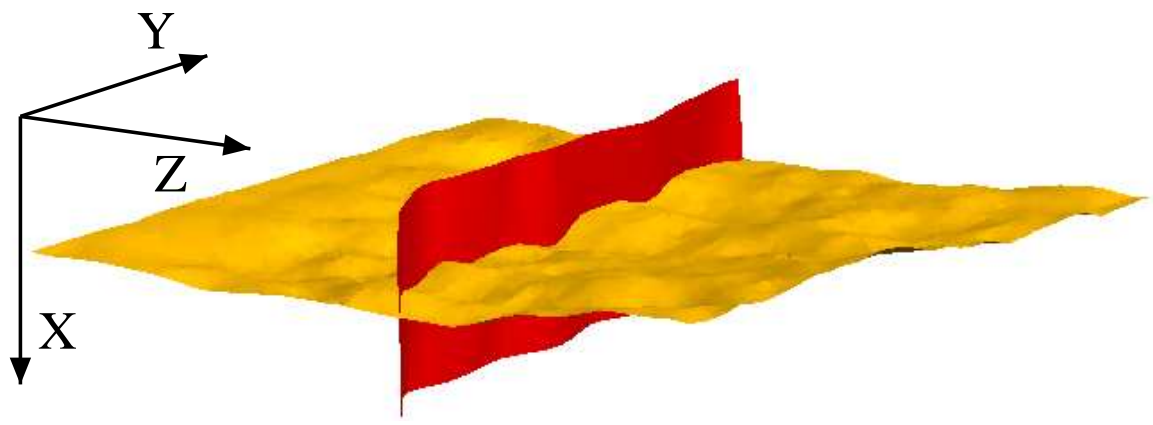

Fig. 19. Crack surface and front level sets obtained in Section 4

In the proposed eXtended Finite Element method, enrichments are introduced in the finite element mesh according to the previous level sets. This allows one to capture the discontinuity and the asymptotic behavior close to the crack front even with a rather coarse mesh. In Figure 20, the three dimensional displacement field obtained with 8-voxel elements is plotted and can be compared to the corresponding one obtained by X3D-DIC (Figure 16).

The main interest of this approach lies in the robustness of the method and its ability to easily take into account a given crack geometry with no modification of the mesh. In this simulation, a linear elastic behavior was considered. Furthermore, an estimate of the 3D plastic zone is obtained from the computed von Mises stress field (Figure 21). However, some remarks can be made, namely, the good agreement observed between the displacement field from linear elastic simulations (Figure 20) and experiments (Figures 3 and 16) together with the small size of the plastic zone allow one to assume confined plasticity, as a good approximation. Hence, a linear mechanical model is considered for the numerical study [61].

The confined plasticity domain is an approximation compared to an elastoplastic calculation. However, the linear model gives a good estimation of the boundary effects close to the crack front and its interaction with the free 

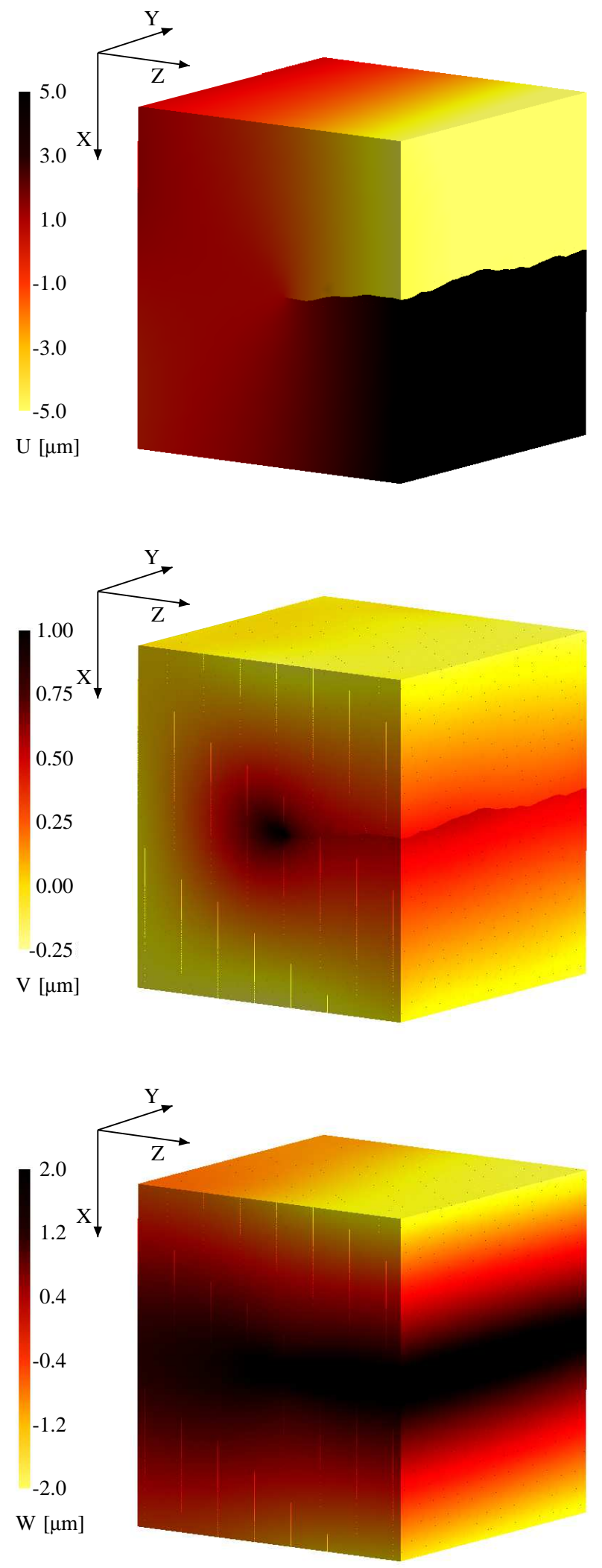

Fig. 20. The three components of X-FEM displacement field (in micrometers) for the maximum load level $(186 \mathrm{~N})$ using the crack geometry detected at 45,000 cycles. 45

The volume shown in this figure is a cube whose edge is $1.46 \mathrm{~mm}$ (or 288 voxel) long. 

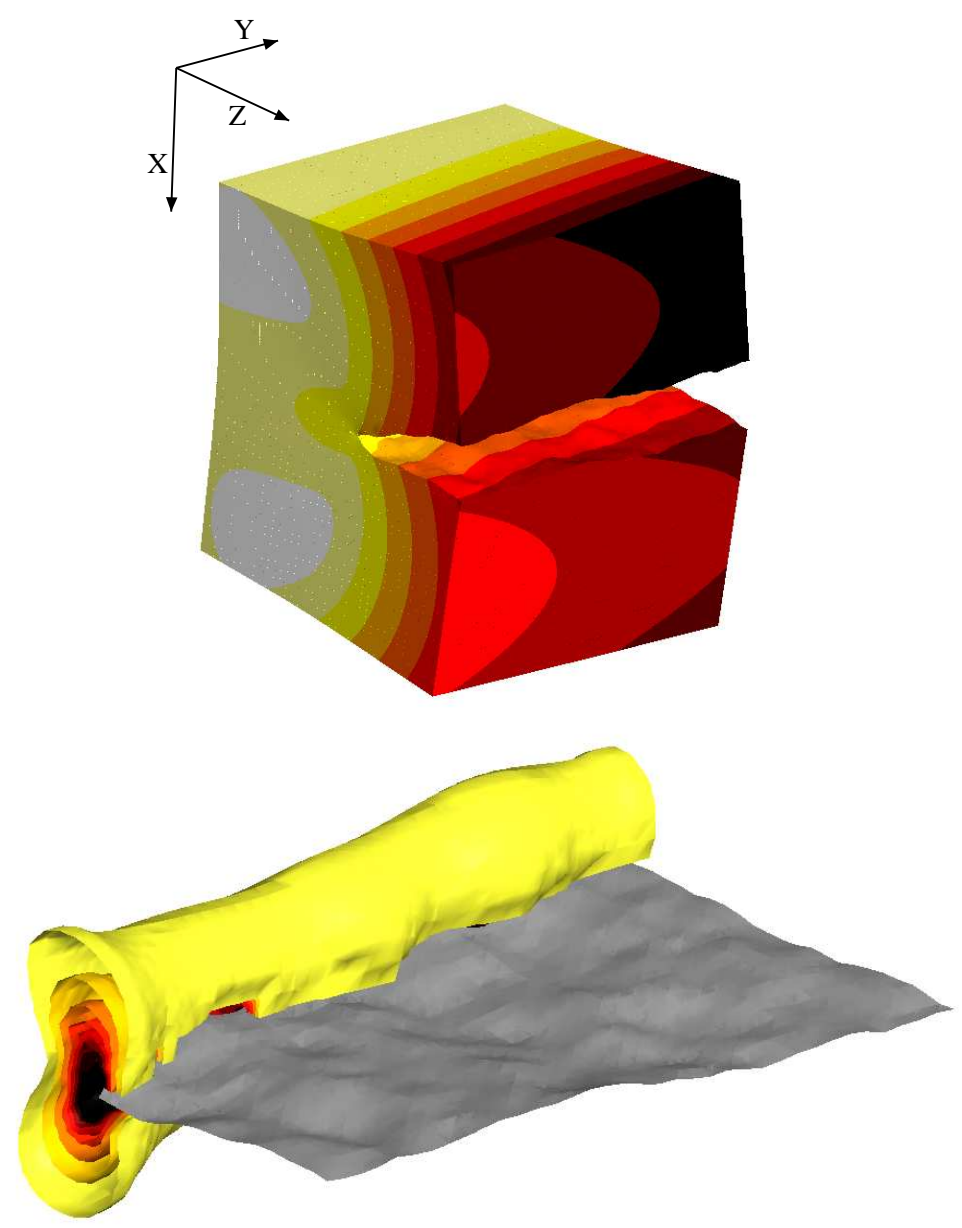

Fig. 21. Deformed configuration and thresholded von Mises stress field around the crack tip for the maximum load level.

surfaces. Furthermore, in a next paragraph, the corresponding stress intensity factors obtained by a domain (and path-independent) integral from the XFEM computations will be compared with experimental values. From a general point of view, one proposes an automatic strategy that allows one to compare displacement fields and stress intensity factors obtained from 3D digital image correlation and 3D simulations with a similar crack geometry. 


\section{SIF estimation from X-DIC and X-FEM}

The following paragraphs concern the computation of stress intensity factors. The proposed approach is general and can be applied to both experimental or numerical displacement fields. In this respect, an extension of a previous study [38] to the three-dimensional case is proposed. A domain integral is used to evaluate the different stress intensity factors from three-dimensional digital image correlation or from elastic X-FEM simulations performed on the same geometry.

\subsection{Methodology}

It is assumed that a set of nodes has been defined (along the experimental or numerical crack front) with their own local basis. A domain integral is considered to compute the energy release rate at any point along the front, and a so-called interaction integral is used to separately compute the three stress intensity factors [62]. For each point along the crack front parameterized by its curvilinear abscissa, $s$, a domain $\mathcal{S}(s)$ is defined in the plane $P(s)$ normal to the crack front and extruded along the crack front in a 3D integration domain $\Omega$. Figure 22(a) shows such 3D domain whose sections are here chosen as circular disks. For any chosen fracture mode, the displacement and stress fields obtained from Westergaard's solution [63] are denoted respectively $u^{\text {aux }}$ and $\sigma^{\text {aux }}$. A virtual crack extension field $\boldsymbol{q}$ that vanishes on the boundary of the integration domain is considered [32]. In addition, this virtual crack extension field is parallel to the crack face, and is a unit vector aligned with the crack direction at the crack tip $\left(\boldsymbol{q}(s)=l_{n}(s) \boldsymbol{x}_{1}(s)\right)$. For each domain $\Omega$ 

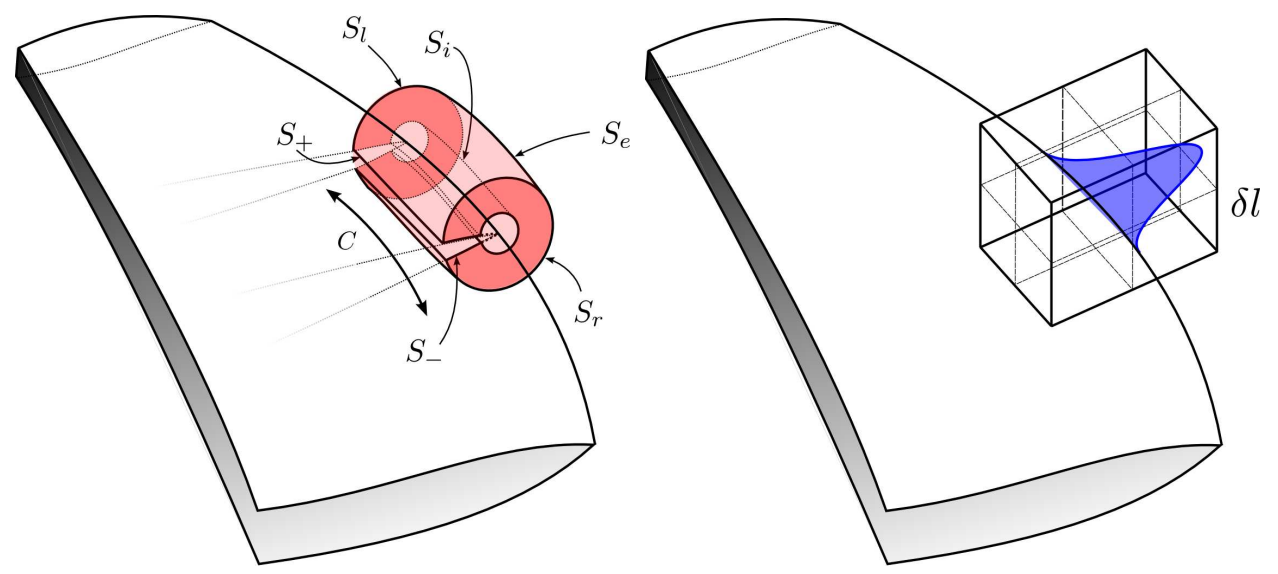

Fig. 22. 3D integration domain and virtual crack extension field along the crack front for the computation of the interaction integral

along the crack front the following interaction integral is computed

$$
I^{\text {int }}=-\int_{\Omega(s)}\left[\sigma_{m l}^{\mathrm{aux}} u_{m, l} \delta_{k j}-\left(\sigma_{i j}^{\mathrm{aux}} u_{i, k}+\sigma_{i j} u_{i, k}^{\mathrm{aux}}\right)\right] q_{k, j} \mathrm{~d} \Omega
$$

and provides local SIFs using Irwin's relationship

$$
\frac{I^{\text {int }}(s)}{\int_{\mathcal{C}} \delta l_{n}(s) \mathrm{d} s}=\frac{2\left(1-\nu^{2}\right)}{E}\left(K_{I} K_{I}^{\mathrm{aux}}+K_{I I} K_{I I}^{\mathrm{aux}}\right)+\frac{2(1+\nu)}{E} K_{I I I} K_{I I I}^{\mathrm{aux}}
$$

where $K_{i}^{\text {aux }}$ are auxiliary stress intensity factors chosen to extract successively the actual mode I, II and III stress intensity factors. The specific interaction integral used herein was introduced in Ref. [64] for planar cracks with curved fronts and in Ref. [65] for non-planar cracks.

The intersection between the crack front and the free surface introduces some difficulties. It is proposed to circumvent them as illustrated in Figure 23 by multiplying the product of the virtual crack extension field and the test function $\delta l(s)$ with a ramp function that vanishes on the free surface. Hence stress intensity factors are not estimated on the surface, although they can be extrapolated from their values in the bulk. For all inner nodes along the crack front, the (discretized) stress intensity factors are obtained with this procedure. 

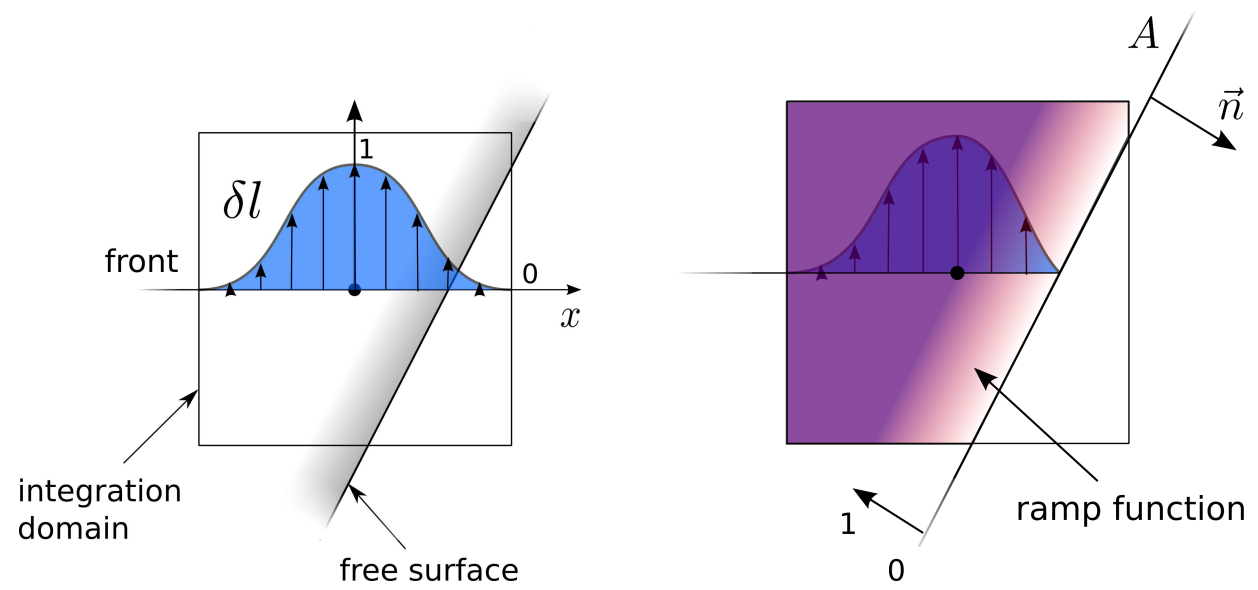

Fig. 23. Specific virtual crack extension field close to a free surface

It should be underlined that the use of such formalism is secure for numerical simulations in elasticity. However, for measured displacement fields (e.g., by DIC), the fact that the equilibrium conditions are not strictly satisfied introduces much more uncertainty. Thus in this context, the SIF determination from the above formalism is to be considered as one among other possibilities. However, the fact that this determination defines an "extractor" field, i.e., a vector field whose scalar product with the displacement field provides the stress intensity factor is a strong property that ensures an unbiased estimate in the presence of measurement uncertainties. Further extensions of this formalism that optimize the noise robustness were designed and tested in two dimensions [39]. However, the transposition to three dimensions is not yet available.

\subsection{Results}

From the experimental displacement fields presented in Section 5, stress intensity factors are computed directly. An integration domain of $5 \times 5 \times 5$ elements of the displacement mesh is used for this computation. As the dis- 
placement is obtained on a finite element mesh with an X-FEM discretization, a numerical simulation is also performed with the experimental mesh, the experimental crack geometry and experimental loadings. Note that the mesh covers the region of interest that has a volume of $288 \times 288 \times 288$ voxels (or $1.46 \times 1.46 \times 1.46 \mu \mathrm{m}^{3}$ ) centered in the tomographic image frame. Stress intensity factors are thus estimated at the nodes inside the $Y$ range from $130 \mu \mathrm{m}$ to $1590 \mu \mathrm{m}$ (in the image coordinates system). Despite some oscillations due to the noisy nature of the displacement field along the crack front (Figure 24) experimental and numerical results are in reasonable agreement. Both numerically and experimentally based stress intensity factors in mode I, $K_{\mathrm{I}}$, are proportional to the applied load. For the maximum load level a mean value of $14 \mathrm{MPa} \sqrt{\mathrm{m}}$ is obtained. It was already reported that the crack front is oblique with respect to the specimen geometry. The two crack lengths measured on the two opposite free edges differ by about $220 \mu \mathrm{m}$, but no significant effect is observed as $K_{\mathrm{I}}$ is nearly independent of the crack length variation. The rather large element size of 32 voxels used in the present case to improve the robustness of the X3D-DIC algorithm does not allow for a fine spatial resolution of the change of the stress intensity factors along the crack front.

Figure 25 shows mode I, II and III stress intensity factors along the crack front. It is observed that mode I is predominant but mode III also develops locally, oscillating from about $8 \mathrm{MPa} \sqrt{\mathrm{m}}$ to $-5 \mathrm{MPa} \sqrt{\mathrm{m}}$ within the region limited by $Y=200 \mu \mathrm{m}$ and $Y=800 \mu \mathrm{m}$. Mode III corresponds to a discontinuity of $V$ as observed in Figure 16.

For comparison purposes, stress intensity factors are also estimated from the elastic analysis carried out in sub-Section 6.3. Figure 26 shows that the tortuosity of the crack front influences the change of the stress intensity factors 


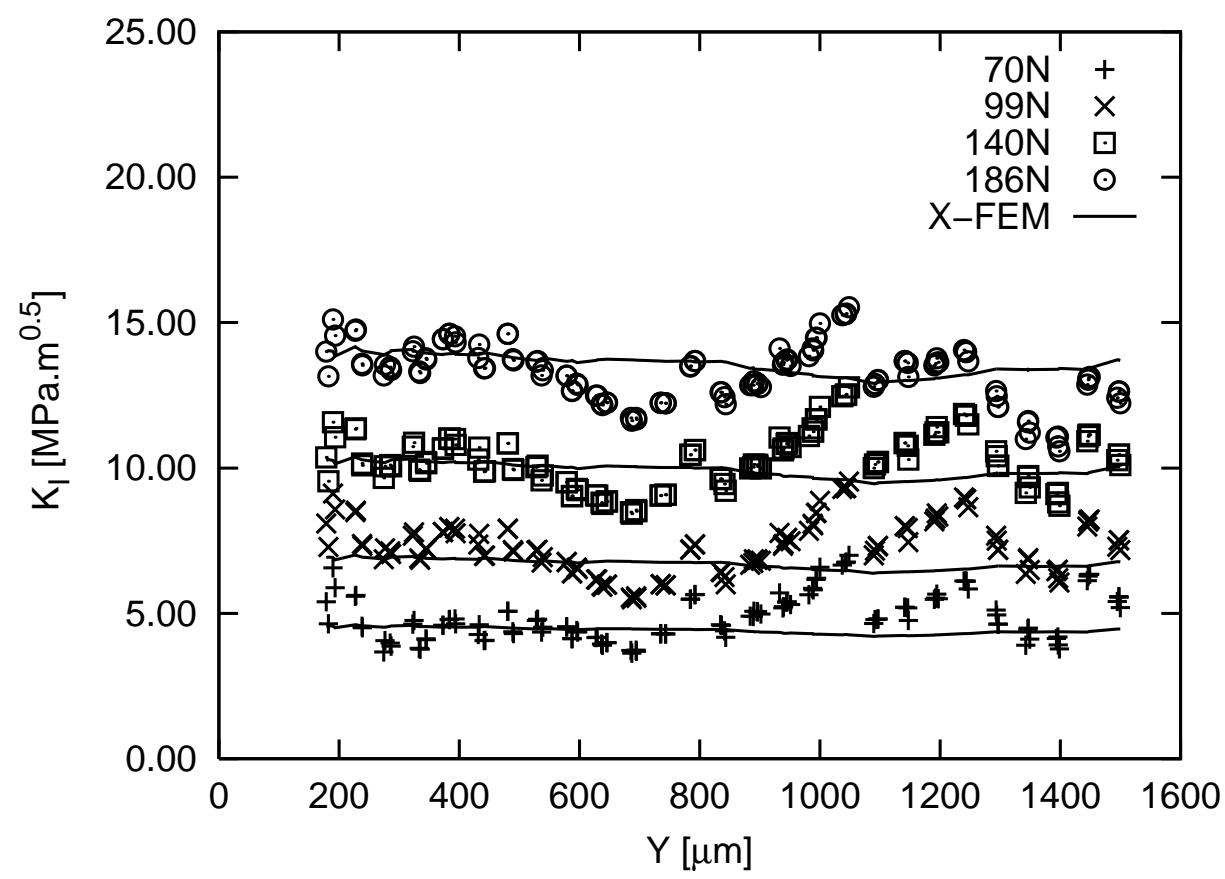

Fig. 24. X-FEM and X-DIC mode I stress intensity factor along the crack front after 45,000 cycles. Experimental results are compared with numerical simulations (X-FEM) on the same mesh, same crack geometry under experimental boundary conditions.

but the average values are consistent.

\section{Discussion}

3D characterizations of cracks in optically opaque materials using synchrotron X-ray microtomography is a recent research area triggered, in the late nineties, by the availability of third generation synchrotrons [66]. Since then, a growing number of papers has been published on that topic (see for example Ref. [42] for a list of studies on metals). In all these works, the characterization of the crack shape was performed by direct analysis (i.e., visual inspection and / or thresholding) of the reconstructed 3D images. However, even at the best resolution available (of the order of $1 \mu \mathrm{m}$ ), crack tip displacements are unlikely to 


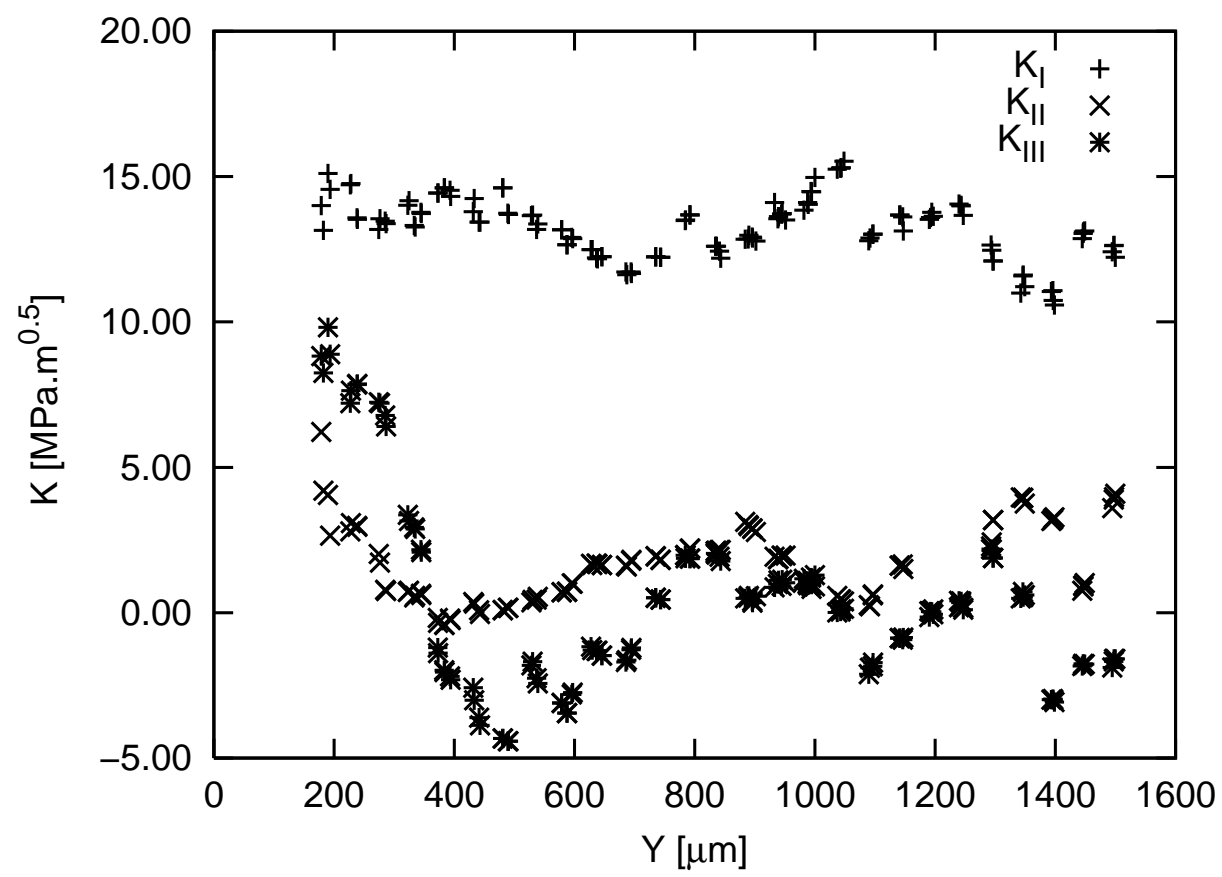

Fig. 25. X-DIC stress intensity factors along the crack front for the maximum load level after 45,000 cycles.

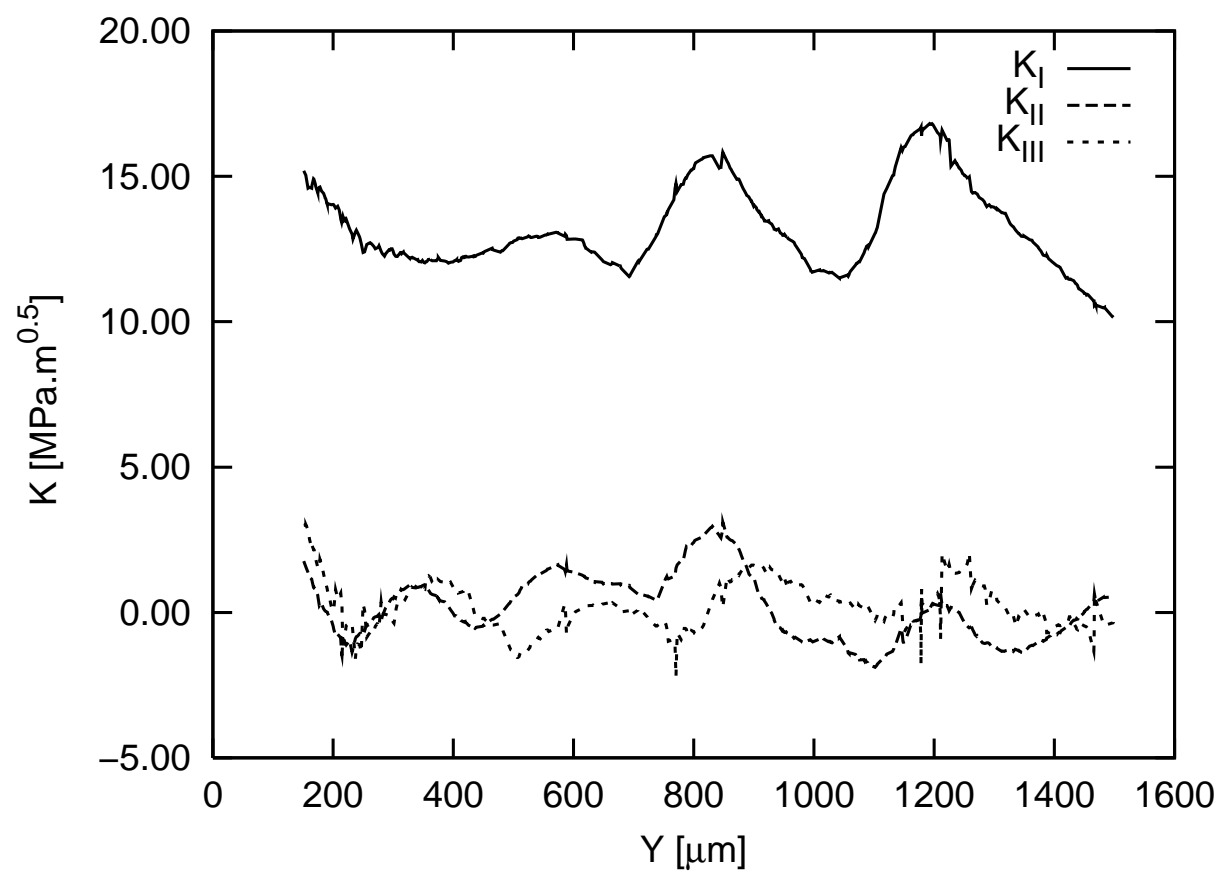

Fig. 26. X-FEM stress intensity factors along the crack front for the maximum load level after 45,000 cycles obtained from the numerical simulation of Section 6.3 
be fully detected by direct inspection of the images. The use of phase contrast available on modern synchrotron sources was shown to increase crack detection to some extent $[15,40]$ but even in that case only a conservative image of the crack can probably be imaged by tomography. DIC has already been used, in a few studies, on tomographic images [24, 28, 67] but not with the explicit purpose of detecting cracks.

The results presented herein show that DIC is a very efficient way of analyzing 3D images of cracked specimens. The 3D shape of the crack surface obtained by using the correlation residuals is in very good agreement with that obtained with direct (i.e., more classical) observations of the images (Figure 6). For the reasons given before, one would have expected the crack detected by DIC to be longer that the one observed directly on the reconstructed images. However, this does not seem to be the case, probably because of the relatively coarse voxel size used $(\sim 5 \mu \mathrm{m})$ that sets a limit to the accuracy with which the residuals are obtained and subsequently thresholded.

Experiments with a smaller voxel size can be considered in the future but the high energy of the incoming X-ray beam required by the large X-ray attenuation of the ferrous matrix sets a limit to the resolution / efficiency of the detector used. A voxel size of $\sim 3 \mu \mathrm{m}$ is currently available on beamline ID19. However, the size of and the distance between the "markers" used herein (i.e., the graphite nodules) leads to yet another limit in terms of discretization. It is believed that the resolution chosen is close to an optimal value in terms of nodule content per finite element. A finer microstructure would be needed if the resolution were finer. The type of studied material is also important when DIC techniques are to be used. The cast iron used herein represents an almost ideal case of a microstructure with many randomly spaced small 
natural markers that are not found, for instance in Al-based alloys.

Besides being an effective way of imaging cracks, DIC also brings in a considerable wealth of information (compared with "simple" 3D crack images) via the determination of the displacement field around the crack upon loading the sample. It was shown that this displacement field can be used efficiently to compute local values of stress intensity factors along the crack front. Those values were also obtained directly from X-FEM / level set calculations (Figure 1) based on a realistic 3D crack (as in Ref. [9]). The fact that both methods yield the same order of magnitude for SIFs (Figure 24) is a promising result. Local discrepancies observed in Figure 24 between X-DIC and X-FEM values are probably again the results of the voxel size used, which decreases the signal to noise ratio in the measured displacement.

Experimentally determined values of SIF along a crack front had been obtained in the past from the analysis of high resolution (voxel size of $0.7 \mu \mathrm{m}$ ) 3D tomographic images $[68,69]$. In that case, small porosities close to the tip of a crack were used as markers for "manual" tracking of the displacement field at the crack tip. Compared to this earlier work, the approach presented herein has the advantage of being fully automatic and much less local. Moreover, the DIC analysis provides sub-voxel displacement values in the micrometer range close to those measured by Toda et al. [68]. It is worth noting that the sample studied herein has a larger cross section (i.e., 3 times larger) than the one used by Toda et al. [68], which paves the way for performing crack closure measurements with an increased confidence. Interestingly, the same authors measured a significant contribution of mode III for the opening of their crack macroscopically loaded in mode I. In the present case it was shown in Figure 16 that the studied crack, in spite of being also loaded in mode I, 
exhibited significant values of $K_{I I I}$. This may be due to the loading device used and / or the local geometry of the crack surface. Further analyses are required to clarify this point. Another approach that may be fruitful for future work is to couple elastic computations and X3D-DIC analyses, in order to seek for the displacement field already in the space of elastic solutions. Such integrated approaches have been followed successfully in the past in two dimensions $[8$, $36,70]$.

\section{Summary}

This paper was dedicated to the use of a fully combined experimental / numerical procedure to deal, in an integrated way, with a cracked sample. The following tools were utilized:

- X-Ray microtomography to visualize various states of a fatigue crack within a volume made of cast iron,

- eXtended 3D Digital Image Correlation (X3D-DIC) to measure in situ displacement fields in the bulk in the presence of a crack, and identify stress intensity factors along the crack front.

- eXtended 3D Finite Element Method to analyze stress intensity factors by considering the experimentally determined crack geometry.

The main experimental challenge is the detection of the crack geometry within the body. The enriched basis used herein proves to be the key concept to allow for the detection of crack surfaces and front positions. This approach has led to the concept of X3D-DIC, which is based on a global approach to volume correlation. The detection is based upon the minimization of the quadratic error between two considered scans by using the sought displacement field 
and its representation on the chosen basis (i.e., continuous and enriched shape functions). Hence the optimal solution of the problem requires a combination of finite element analyses and image processing techniques, which ignore the mechanical content of the information. This approach could also be validated by using a pure image processing technique.

The finite element tools are also used directly to post-process the measured displacement field (including the singular components) to extract stress intensity factors along the crack front. The latters are reasonably close to those determined directly from the analysis of the measured displacements.

This type of study opens the way for estimating 3D crack growth experimentally by using a series of analyses as the one presented herein. This may then allow for an identification and even a validation of 3D crack propagation models.

\section{Acknowledgments}

This work was funded by the CETIM Foundation grant entitled PROPAVANFIS: "Advanced methods for the experimental and numerical analyses of crack propagations under complex loadings." This work was also made possible by an ESRF grant for the experiment MA-501 on beamline ID19.

\section{References}

[1] F. Erdogan, Fracture mechanics, Int. J. Solids Struct. 37 [1-2] (2000) 171-183. 
[2] W. G. Knauss, Perspectives in experimental mechanics, Int. J. Solids Struct. 37 (2000) 251-266.

[3] A. J. Rosakis and G. Ravichandran, Dynamic failure mechanics, Int. J. Solids Struct. 37 [1-2] (2000) 331-348.

[4] J. T. Oden, T. Belytschko, I. Babuska and T. J. R. Hughes, Research directions in computational mechanics, Comp. Meth. Appl. Mech. Eng. 192 (2003) 913-922.

[5] M. E. Kassner, S. Nemat-Nasser, Z. Suo, G. Bao, J. C. Barbour, L. C. Brinson, H. Espinosa, H. Gao, S. Granick, P. Gumbsch, K.-S. Kim, W. Knauss, L. Kubin, J. Langer, B. C. Larson, L. Mahadevan, A. Majumdar, S. Torquato and F. van Swol, New directions in mechanics, Mech. Mat. 37 [2-3] (2005) 231-259.

[6] W. Elber, Fatigue Crack Closure under Cyclic Tension, Eng. Fract. Mech. 2 (1970) 37-45. See also W. Elber, The Significance of Fatigue Crack Closure, in: Damage Tolerance in Aircraft Structures (ASTM, STP 486, Philadelphia, 1971), 230-242.

[7] M. A. Sutton, W. Zhao, S. R. McNeill, J. D. Helm, R. S. Piascik and W. T. Riddel, Local crack closure measurements: Development of a measurement system using computer vision and a far-field microscope, in: Advances in fatigue crack closure measurement and analysis: second volume, STP 1343, R. C. McClung and J. C. Newman Jr., eds., (ASTM, 1999), 145-156. See also W. T. Riddel, R. S. Piascik, M. A. Sutton, W. Zhao, S. R. McNeill and J. D. Helm, Determining fatigue crack opening loads from near-crack-tip displacement measurements, in: Advances in fatigue crack closure measurement and analysis: second volume, STP 1343, R. C. McClung and J. Newman, J.C., eds., (ASTM, 1999), 157-174.

[8] R. Hamam, F. Hild and S. Roux, Stress intensity factor gauging by digital 
image correlation: Application in cyclic fatigue, Strain 43 (2007) 181-192.

[9] E. Ferrié, J.-Y. Buffière, W. Ludwig, A. Gravouil and L. Edwards, Threedimensional study of fatigue crack propagation of a semi-elliptical crack: 3D crack visualization using X-ray microtomography and numerical simulation of crack propagation using the Extended Finite Element method, Acta Mat. 54 [4] (2006) 1111-1122.

[10] T. L. Wolfsdorf, W. H. Bender and P. W. Voorhees, The morphology of high volume fraction solid-liquid mixtures: An application of microstructural tomography, Acta Mater. 45 [6] (1997) 2279-2295.

[11] J. Baruchel, J.-Y. Buffière, E. Maire, P. Merle and G. Peix, X-Ray Tomography in Material Sciences, (Hermes Science, Paris (France), 2000).

[12] O. Ludwig, M. Dimichiel, L. Salvo, M. Suéry and P. Falus, In-situ ThreeDimensional Microstructural Investigation of Solidification of an $\mathrm{Al}-\mathrm{Cu}$ Alloy by Ultrafast X-ray Microtomography, Metall. Mat. Trans. A 36 [6] (2005) 1515-1523.

[13] A. Vagnon, O. Lame, D. Bouvard, M. Di Michiel, D. Bellet and G. Kapelski, Deformation of steel powder compacts during sintering: Correlation between macroscopic measurement and in situ microtomography analysis, Acta Mater. 54 [2] (2006) 513-522.

[14] D. Bernard, D. Gendron, J.-M. Heintz, S. Bordere and J. Etorneau, 2005, First direct $3 \mathrm{D}$ visualisation of microstructural evolutions during sintering through X-ray computed microtomography, Acta Mater., 53 121-128.

[15] J.-Y. Buffière, E. Maire, P. Cloetens, G. Lormand and R. Fougères, Characterisation of internal damage in a MMCp using X-ray synchrotron phase contrast microtomography, Acta Mater. 47 (1999) 1613-1625.

[16] L. Babout, E. Maire, J.-Y. Buffière and R. Fougères, Characterisation by X-Ray computed tomography of decohesion, porosity growth and coales- 
cence in model metal matrix composites, Acta Mater. 49 (2001) 20552063.

[17] P. Viot and D. Bernard, Impact test deformations of polypropylene foam samples followed by microtomography, J. Mater. Sci. 41 (2006) 12771279.

[18] P. Viot, D. Bernard and E. Plougonven, Polymeric foam deformation under dynamic loading by the use of the microtomographic technique, $J$. Mater. Sci. 42 [17] (2007) 7202-7213.

[19] S. Youssef, E. Maire and R. Gaertner, Finite element modelling of the actual structure of cellular materials determined by X-ray tomography, Acta Mater. 53 [3] (2005) 719-730.

[20] M. A. Sutton, S. R. McNeill, J. D. Helm and Y. J. Chao, Advances in Two-Dimensional and Three-Dimensional Computer Vision, in: Photomechanics, P. K. Rastogi, eds., (Springer, Berlin (Germany), 2000), 323-372.

[21] P. Cheng, M. A. Sutton, H. W. Schreier and S. R. McNeill, Full-field Speckle Pattern Image Correlation with B-Spline Deformation Function, Exp. Mech. 42 [3] (2002) 344-352.

[22] B. K. Bay, T. S. Smith, D. P. Fyhrie and M. Saad, Digital volume correlation: three-dimensional strain mapping using X-ray tomography, Exp. Mech. 39 (1999) 217-226.

[23] T. S. Smith, B. K. Bay and M. M. Rashid, Digital volume correlation including rotational degrees of freedom during minimization, Exp. Mech. 42 [3] (2002) 272-278.

[24] M. Bornert, J.-M. Chaix, P. Doumalin, J.-C. Dupré, T. Fournel, D. Jeulin, E. Maire, M. Moreaud and H. Moulinec, Mesure tridimensionnelle de champs cinématiques par imagerie volumique pour l'analyse des matériaux et des structures, Inst. Mes. Métrol. 4 (2004) 43-88. 
[25] E. Verhulp, B. van Rietbergen and R. Huiskes, A three-dimensional digital image correlation technique for strain measurements in microstructures, J. Biomech. 37 [9] (2004) 1313-1320.

[26] G. Besnard, F. Hild and S. Roux, "Finite-element" displacement fields analysis from digital images: Application to Portevin-Le Châtelier bands, Exp. Mech. 46 (2006) 789-803.

[27] F. Hild and S. Roux, Digital image correlation: from measurement to identification of elastic properties - A review, Strain 42 (2006) 69-80.

[28] S. Roux, F. Hild, P. Viot and D. Bernard, Three dimensional image correlation from X-Ray computed tomography of solid foam, Comp. Part A 39 [8] (2008) 1253-1265.

[29] N. Moës, J. Dolbow and T. Belytschko, A finite element method for crack growth without remeshing, Int. J. Num. Meth. Eng. 46 [1] (1999) 131150.

[30] G. Zi and T. Belytschko, New crack-tip elements for XFEM and applications to cohesive cracks, Int. J. Num. Meth. Eng. 57 [15] (2003) 2221-2240.

[31] N. Sukumar, N. Moës, B. Moran and T. Belytschko, Extended finite element method for three-dimensional crack modeling, Int. J. Num. Meth. Eng. 48 [11] (2000) 1549-1570.

[32] N. Moës, A. Gravouil and T. Belytschko, Non-planar 3D crack growth by the extended finite element and level sets. Part I: Mechanical model, Int. J. Num. Meth. Eng. 53 [11] (2002) 2549-2568.

[33] J. Réthoré, F. Hild and S. Roux, Shear-band capturing using a multiscale extended digital image correlation technique, Comp. Meth. Appl. Mech. Eng. 196 [49-52] (2007) 5016-5030.

[34] J. Réthoré, F. Hild and S. Roux, Extended digital image correlation with 
crack shape optimization, Int. J. Num. Meth. Eng. 73 [2] (2008) 248-272.

[35] S. R. McNeill, W. H. Peters and M. A. Sutton, Estimation of stress intensity factor by digital image correlation, Eng. Fract. Mech. 28 [1] (1987) 101-112.

[36] S. Roux and F. Hild, Stress intensity factor measurements from digital image correlation: post-processing and integrated approaches, Int. J. Fract. 140 [1-4] (2006) 141-157.

[37] J. Rannou, A. Gravouil and M.C. Baietto-Dubourg, A local multigrid X-FEM stratefy for 3-D crack propagation, Int. J. Num. Meth. Eng. 77 [4] (2009) 581-600.

[38] J. Réthoré, A. Gravouil, F. Morestin and A. Combescure, Estimation of mixed-mode stress intensity factors using digital image correlation and an interaction integral, Int. J. Fract. 132 (2005) 65-79.

[39] J. Réthoré, S. Roux and F. Hild, Noise-robust Stress Intensity Factor Determination from Kinematic Field Measurements, Eng. Fract. Mech. 75 [13] (2008) 3763-3781.

[40] P. Cloetens, M. Pateyron-Salomé, J-Y. Buffière, G. Peix, J. Baruchel and F. Peyrin, Observation of microstructure and damage in materials by phase sensitive radiography, J. Appl. Phys. D, 81 [9] (1997) 5878-5886.

[41] J.-C. Labiche, O. Mathon, S. Pascarelli, M. A. Newton, G. Guilera Ferre, C. Curfs, G. Vaughan, A. Homs and D. Fernandez Carreiras, The fast readout low noise camera as a versatile X-ray detector for time resolved dispersive extended X-ray absorption fine structure and diffraction studies of dynamic problems in materials science, chemistry and catalysis, Rev. Sci. Instrum. 78 [091301] (2007).

[42] J.-Y. Buffière, E. Ferrié, H. Proudhon, W. Ludwig, Three-dimensional visualisation of fatigue cracks in metals using high resolution synchrotron 
X-ray micro-tomography Mat. Sci. Tech. 22 [9] (2006) 1019-1024.

[43] A. C. Kak and M. Slaney, Principles of Computerized Tomographic Imaging, Society of Industrial and Applied Mathematics (2001).

[44] S. F. Nielsen, H. F. Poulsen, F. Beckmann, C. Thorning and J. A. Wert, Measurements of plastic displacement gradient components in three dimensions using marker particles and synchrotron X-ray absorption microtomography, Acta Mater. 51 [8] (2003) 2407-2415.

[45] B. K. Bay, Texture correlation: A method for the measurement of detailed strain distributions within trabecular bone, J. Orthopaedic Res. 13 [2] (1995) 258-267.

[46] T. O. McKinley and B. K. Bay, Trabecular bone strain changes associated with subchondral stiffening of the proximal tibia, J. Biomech. 36 [2] (2003) 155163.

[47] N. Lenoir, M. Bornert, J. Desrues, P. Bésuelle and G. Viggiani, Volumetric digital image correlation applied to X-ray microtomography images from triaxial compression tests on argillaceous rock, Strain 43 (2007) 193-205.

[48] H. Bart-Smith, A.-F. Bastawros, D. R. Mumm, A. G. Evans, D. J. Sypeck and H. N. G. Wadley, Compressive deformation and yielding mechanisms in cellular $\mathrm{Al}$ alloys determined using X-ray tomography and surface strain mapping, Acta Mater. 46 [10] (1998) 3583-3592.

[49] F. Hild, E. Maire, S. Roux and J.-F. Witz, Three dimensional analysis of a compression test on stone wool, Acta Mat. [DOI: 10.1016/j.actamat.2009.03.038] (2009).

[50] F. Hild, B. Raka, M. Baudequin, S. Roux and F. Cantelaube, Multi-Scale Displacement Field Measurements of Compressed Mineral Wool Samples by Digital Image Correlation, Appl. Optics IP 41 [32] (2002) 6815-6828.

[51] I. Babuska and J. M. Melenk, The partition of unity method, Int. J. Num. 
Meth. Eng. 40 [4] (1997) 727758.

[52] http://www.amiravis.com/.

[53] http://rsb.info.nih.gov/ij/.

[54] J. A. Sethian, Level set methods and fast marching methods. Cambridge University Press, 1999.

[55] A. Gravouil, N. Moës and T. Belytschko, Non-planar 3D crack growth by the extended finite element and level sets. Part II: Level set update, Int. J. Num. Meth. Eng. 53 [11] (2002) 25492568.

[56] M. Duflot, A study of the representation of cracks with level sets, Int. J. Num. Meth. Eng. 70 [11] (2006) 1261-1302.

[57] P. A. Guidault, O. Allix, L. Champaney, J. P. Navarro, A multiscale computational strategy for crack propagation with local enrichment, Comp. Struct. 85 [17-18] (2007) 1360-1371.

[58] A. Brandt, Multi-level adaptive solutions to boundary-value problems, Math. of Comp. 31 [138] (1977) 333-390.

[59] I. D. Parsons, J. F. Hall, The multigrid method in solid mechanics: Part Ialgorithm description and behaviour, Int. J. Num. Meth. Eng. 29 (1990) 719-737.

[60] J. Fish, M. Pandheeradi and V. Belsky, An efficient multilevel solution scheme for large scale non-linear systems, Int. J. Num. Meth. Eng. 38 (1995) 1597-1610.

[61] T. Elguedj, A. Gravouil, A. Combescure, Appropriate extended functions for X-FEM simulation of plastic fracture mechanics, Comp. Meth. Appl. Mech. Eng. 195 [7-8] (2006) 501-515.

[62] E. Béchet, H. Minnebo, N. Moës and B. Burgardt, Improved implementation and robustness study of the X-FEM for stress analysis around cracks, Int. J. Num. Meth. Eng. 64 (2005) 1033-1056. 
[63] H. M. Westergaard, Bearing pressures and cracks, J. Appl. Mech. 6 (1939) A49A53.

[64] M. Gosz, J. Dolbow and B. Moran, Domain integral formulation for stress intensity factor computation along curved three-dimensional interface cracks, Int. J. Solids Struct. 35 [15] (1998) 1763-1783.

[65] M. Gosz, J. Dolbow and B. Moran, An interaction energy integral method for computation of mixed-mode stress intensity factors along non-planar crack fronts in three dimensions, Eng. Fract. Mech. 69 [3] (2002) 299-319.

[66] A. Guvenilir, T. M. Breunig, J. H. Kinney and S. R. Stock, Direct observation of crack opening as a function of applied load in the interior of a notched tensile sample of Al-Li 2090 Acta Mat. 45 [5] 1977-1987.

[67] M. Kobayashi, H. Toda, Y. Kawai, T. Ohgaki, K. Uesugi, D. S. Wilkinson, T. Kobayashi, Y. Aoki and M. Nakazawa, High-density three-dimensional mapping of internal strain by tracking microstructural features Acta Mat. 56 [10] (2008) 2167-2181.

[68] H. Toda, I. Sinclair, J.-Y. Buffière, E. Maire, K. H. Khor, P. Gregson and T. Kobayashi, A 3D measurement procedure for internal local crack driving forces via synchrotron X-ray microtomography Acta Mat. 52 [5] (2004) 1305-1317.

[69] H. Toda, I. Sinclair, J.-Y. Buffière, E. Maire, T. Connolley, M. Joyce, K. H. Khor and P. Gregson, Assessment of the fatigue crack closure phenomenon in damage-tolerant aluminium alloy by in-situ high-resolution synchrotron X-ray microtomography, Phil. Mag. 83 [21] (2003) 2429-2448.

[70] J. Réthoré, S. Roux and F. Hild, An extended and integrated digital image correlation technique applied to the analysis fractured samples, Eur. J. Comput. Mech. 18 (2009) 285-306. 